\title{
Cross-correlation search for periodic gravitational waves
}

\author{
Sanjeev Dhurandhar, ${ }^{2, *}$ Badri Krishnan, ${ }^{1, \dagger}$ Himan Mukhopadhyay, ${ }^{2}$ and John T. Whelan ${ }^{1, \ddagger}$ \\ ${ }^{1}$ Max-Planck-Institut für Gravitationsphysik, Albert-Einstein-Institut, Am Mühlenberg 1, D-14476 Golm, Germany \\ ${ }^{2}$ Inter-University Centre for Astronomy and Astrophysics, Post Bag 4, Ganeshkhind, Pune 411007, India
}

(Received 18 December 2007; published 17 April 2008)

\begin{abstract}
In this paper we study the use of cross correlations between multiple gravitational wave (GW) data streams for detecting long-lived periodic signals. Cross-correlation searches between data from multiple detectors have traditionally been used to search for stochastic GW signals, but recently they have also been used in directed searches for periodic GWs. Here we further adapt the cross-correlation statistic for periodic GW searches by taking into account both the nonstationarity and the long-term-phase coherence of the signal. We study the statistical properties and sensitivity of this search and its relation to existing periodic wave searches, and describe the precise way in which the cross-correlation statistic interpolates between semicoherent and fully coherent methods. Depending on the maximum duration over which we wish to preserve phase coherence, the cross-correlation statistic can be tuned to go from a standard crosscorrelation statistic using data from distinct detectors, to the semicoherent time-frequency methods with increasing coherent time baselines, and all the way to a full coherent search. This leads to a unified framework for studying periodic wave searches and can be used to make informed trade-offs between computational cost, sensitivity, and robustness against signal uncertainties.
\end{abstract}

DOI: 10.1103/PhysRevD.77.082001

PACS numbers: 04.80.Nn, 04.30.Db, 07.05.Kf, 95.55.Ym

\section{INTRODUCTION}

Long-lived quasiperiodic gravitational waves (GWs) from rapidly rotating nonaxisymmetric neutron stars are among the promising sources of detectable GWs for ground-based detectors such as LIGO, Virgo, GEO600, etc. A number of searches for long-lived periodic GWs have been carried out using data from ground-based GW detectors. These include searches using data from interferometric and bar detectors. These searches are of two kinds depending on the size of the parameter space that is searched:

(i) Targeted searches for sources whose parameters are well known from other astrophysical observations [1-3]. Such searches are not computationally intensive, and use statistically optimal matched filtering techniques.

(ii) Wide parameter space searches either for neutron stars in binary systems whose parameters are poorly constrained from prior observations [4], or blind searches for as yet unknown neutron stars [4-8].

While none of the above searches have yet resulted in a detection, there have been some notable successes. For the searches targeting known pulsars, the limits on the gravitational wave emission and the corresponding limits on the deformation are starting to become astrophysically interesting.

Similarly, a lot of the groundwork has been laid for meeting the computational challenges for the wide parameter space searches. Computationally efficient methods and

\footnotetext{
*sanjeev@iucaa.ernet.in

†badri.krishnan@aei.mpg.de

¥john.whelan@aei.mpg.de
}

hierarchical data analysis pipelines have been developed which allow us to vastly improve the ratio of sensitivity to computational cost. Most of these are semicoherent methods, i.e. combinations of coherent analyses combined together by excess-power techniques, and they come in two main flavors. The first combines short segments of simple Fourier transformed data. The baseline of the short Fourier transforms is chosen such that the signal manifests itself as excess power in a single frequency bin, and the excess power is combined by various methods. The simplest is the StackSlide method [9] which adds the normalized excess power from the short segments, taking care to "slide" the frequency bins to account for the Doppler shift and intrinsic spin-down. The PowerFlux method [10] is very similar; it performs a weighted sum of the normalized power using weights which take the sky position and polarization dependent sensitivity of the detector into account; the weights serve to improve the sensitivity. Finally, there is the Hough transform method $[5,6,11]$ which performs a weighted sum of binary-number counts calculated by setting a threshold on the normalized excess power. This is more robust and computationally efficient, though at the cost of being somewhat less sensitive. All three methods have been used to analyze LIGO data in all-sky wide frequency band searches for GWs from isolated neutron stars [5,6], and these are so far the most sensitive wide parameter space GW searches of their kind published so far; we shall refer to them as the "standard" semicoherent searches in the rest of this paper.

A variant of these standard semicoherent techniques are the so-called hierarchical searches which aim to search deeper by increasing the coherent time baseline $[9,11,12]$. This requires a sky-position (and spin-down) dependent demodulation to be performed before calculat- 
ing the excess-power statistic. The extra demodulation step significantly increases the computational cost and such a search pipeline is currently being employed on larger computational platforms such as EINSTEIN@HOME [13].

In addition to the above surveys for isolated neutron stars, searches have also been carried out for gravitational waves from neutron stars in binary systems. A plausible argument for why some neutron stars may be emitting detectable GWs applies to neutron stars in binary systems, and, in particular, to the low mass x-ray binaries (LMXBs) which consist of a neutron star and a low mass mainsequence star. The observed x-ray flux from these systems is due to the high rates of accretion of matter onto the neutron star. It is observed that the rotation rates of neutron stars in LMXBs is significantly lower than what might be expected on theoretical grounds; the highest theoretically possible rotation rate is significantly larger than $1 \mathrm{kHz}$, while the current observed record is $\sim 620 \mathrm{~Hz}$. It was suggested (first by Bildsten [14]) that this apparent upper bound on the rotation rate might be due to a balance between the spin-up due to accretion and the spin-down due to the emission of gravitational radiation - there is virtually a "wall" created by the flux of GW radiated, which increases as $\Omega^{6}$, where $\Omega$ is the angular rotational frequency of the spinning neutron star and this limits its spin-up. There are a number of other suggested explanations which do not involve gravitational radiation, but accreting neutron stars are clearly promising sources of detectable gravitational radiation. So far, two searches have targeted Sco X-1, the brightest LMXB. These have used very different techniques; [4] used a coherent integration on 6 hours of data from the second science run of the LIGO detectors, while [7] uses a cross-correlation statistic on data from the more recent fourth science run. The elucidation and generalization of this cross-correlation technique tailored to periodic GW searches, and its relation with the other searches discussed above, will occupy us for the rest of this paper.

Using data from the latest science runs of the LIGO detectors, it is expected that the indirect spin-down limit on the amplitude of gravitational waves from the Crab pulsar will be beaten by about a factor of 3 . The resulting limits on the ellipticity of the known pulsars are also starting to place constraints on the equations of state of nuclear matter in neutron stars (see e.g. $[15,16]$ ). A detection would lead to new insights about neutron star physics not obtainable by other means. Searches using large amounts of data from the LIGO detectors operating at design sensitivity are well underway.

Almost all of these searches mentioned above have been based on techniques which look for signals of a given form in a single data stream, i.e. either matched filtering techniques or semicoherent power summing methods. While both matched filtering and semicoherent techniques have been generalized and used to analyze data from multiple interferometers $[6,17]$, the starting point for these methods is always the analysis of a single data stream. There is however one exception, which is the method used in $[7,18]$ and is inherently based on looking at multiple independent data streams. Let us consider two data segments:

$$
\begin{aligned}
& x_{1}(t) \text { for } t \in\left[T_{1}-\Delta T / 2, T_{1}+\Delta T / 2\right], \\
& x_{2}(t) \text { for } t \in\left[T_{2}-\Delta T / 2, T_{2}+\Delta T / 2\right] .
\end{aligned}
$$

The two independent data streams $x_{1,2}$ are typically from different detectors, but they could also be from the same detector for nonoverlapping time intervals. If a signal resulting from the same gravitational wave source is present in both streams (and if the signal is expected to be phase coherent across the two time intervals), it should be possible to cross correlate the output of two detectors to extract the signal. The basic cross-correlation statistic is

$$
\int_{T_{1}-\Delta T / 2}^{T_{1}+\Delta T / 2} d t_{1} \int_{T_{2}-\Delta T / 2}^{T_{2}+\Delta T / 2} d t_{2} x_{1}\left(t_{1}\right) x_{2}\left(t_{2}\right) Q\left(t_{1}, t_{2}\right),
$$

where $Q\left(t_{1}, t_{2}\right)$ is an appropriately chosen filter function. This technique was originally developed for the stochastic background searches where the cross correlation is absolutely essential and is based on the fact that multiple detectors will see the same GW signal $[19,20]$, and it has been used extensively to search for a stochastic GW background using LIGO data [21-23]. The function $Q\left(t_{1}, t_{2}\right)$ can be tuned to search for GWs coming from a particular sky position and also polarization [20] and this method has been used to search for periodic waves from the neutron star in Sco X-1. All previous discussions of this method have however been in the context of stochastic searches. In this paper, we investigate in detail its applications for periodic wave searches.

The optimal form of the function $Q\left(t_{1}, t_{2}\right)$ depends on the kinds of sources that we are looking for. Thus, for a stochastic background we use the facts that the statistical properties of the signal are time independent and that the two polarizations are statistically independent. In particular, the optimal $Q$ is time invariant, i.e. a function of only the difference $t_{1}-t_{2}$. Furthermore, $Q$ turns out to depend on the expected spectrum of the stochastic background.

For periodic GWs from neutron stars, many of these assumptions do not hold. The signal is deterministic and nonstationary (because of the Doppler shift), and the two polarizations are not independent. There is yet another ingredient present for periodic signals that is not present for stochastic sources. In principle, since the signals we are looking for have long-term-phase coherence, it should be possible to cross correlate any pair of data segments to extract the signal, regardless of how far apart the segments are in time and regardless of whether they are from the same interferometer or not. It will turn out that the sky resolution is much coarser than for the standard periodic searches; the appropriate baseline is not the Earth-Sun distance but rather the distance between the two detectors. 
This leads to a much lighter computational burden for a blind search. All of these issues will be discussed in detail in the rest of this paper.

The paper is organized as follows. Section II sets up notation and describes the waveforms that we are looking for; this includes both isolated neutron stars and neutron stars in binary systems. It also discusses the short segment Fourier transforms (SFTs) and the restrictions on their time baseline for the signal power to be concentrated in a single SFT frequency bin. Section III motivates and defines the basic cross-correlation statistic for a pair of short data segments; Sec. IV discusses the statistical properties and the sensitivity of the search; Sec. V elucidates the relationship of the cross-correlation method to the $\mathcal{F}$ statistic; Sec. VI provides estimates of the parameter estimation that can be achieved and Sec. VII investigates the question of resolution of parameters such as sky position, spindown, etc. Section VIII concludes with a summary of our results and suggestions for future work, and finally the Appendix discusses some technical and conceptual issues which have been ignored in the earlier sections for simplicity.

\section{NOTATION AND USEFUL EQUATIONS}

\section{A. The waveform}

The waveform we are looking for is a tensor metric perturbation,

$$
\overleftrightarrow{h}(t)=h_{+}(t) \stackrel{\leftrightarrow}{e}_{+}+h_{\times}(t) \overleftrightarrow{e}_{\times}
$$

where $\left\{\overleftrightarrow{e}_{A} \mid A=+, \times\right\}$ is a transverse-traceless polarization basis associated with the GW propagation direction and tailored to the polarization state of the waves so that

$$
h_{+}(t)=A_{+} \cos \Phi(t), \quad h_{\times}(t)=A_{\times} \sin \Phi(t) .
$$

If $\iota$ is the angle between the line of sight $\vec{n}$ to the star and its rotation axis, the amplitudes are

$$
\begin{array}{rr}
A_{+}=h_{0} \mathcal{A}_{+}, & A_{\times}=h_{0} \mathcal{A}_{\times},(2.3 \mathrm{a}) \\
\mathcal{A}_{+}=\frac{1+\cos ^{2} \iota}{2}, & \mathcal{A}_{\times}=\cos \iota .(2.3 \mathrm{~b})
\end{array}
$$

In the neutron star rest frame with proper time $\tau$, the phase is

$$
\Phi(t(\tau))=\Phi_{0}+2 \pi\left\{f_{0} \tau+\frac{1}{2} f_{1} \tau^{2} \ldots\right\} .
$$

The reference time where all the spin-down parameters are defined is taken to be $\tau=0$, and $\Phi_{0}$ is the phase at $\tau=0$.

A detector's scalar strain response is the contraction of the tensor metric perturbation with a response tensor ${ }^{1} \stackrel{\leftrightarrow}{d}$ :

\footnotetext{
${ }^{1}$ Eor an interferometer with arms along the unit vectors $\vec{u}$ and $\vec{v}, d=\frac{1}{2}(\vec{u} \otimes \vec{u}-\vec{v} \otimes \vec{v})$.
}

$$
h(t)=\stackrel{\leftrightarrow}{h}(t): \stackrel{\leftrightarrow}{d}(t)=\sum_{A=+, \times} F_{A}(t) h_{A}(t)
$$

where

$$
F_{A}(t)=\stackrel{\leftrightarrow}{e}_{A}: \stackrel{\leftrightarrow}{d}(t)
$$

The polarization basis $\left\{\vec{e}_{A}\right\}$ is sometimes inconvenient, because its definition involves not only the direction to the source but also the source's polarization state (specifically the orientation of the neutron star's spin). For a given sky direction $\vec{n}$, one can always construct a transverse, traceless polarization basis $\stackrel{\leftrightarrow}{\varepsilon}_{A}$ by starting e.g., with the vector transverse to $\vec{n}$ and lying in the Earth's equatorial plane. The relationship between this reference basis and the preferred polarization basis of the source is described by the polarization angle $\psi$ :

$$
\begin{aligned}
& \stackrel{\leftrightarrow}{e}_{+}=\overleftrightarrow{\varepsilon}_{+} \cos 2 \psi+\overleftrightarrow{\varepsilon}_{\times} \sin 2 \psi \\
& \overleftrightarrow{e}_{\times}=-\overleftrightarrow{\varepsilon}_{+} \sin 2 \psi+\stackrel{\leftrightarrow}{\varepsilon}_{\times} \cos 2 \psi
\end{aligned}
$$

That means, if we define

$$
\begin{aligned}
& a(t ; \vec{n})=\stackrel{\leftrightarrow}{d}(t): \stackrel{\leftrightarrow}{\varepsilon}_{+}(\vec{n}) \\
& b(t ; \vec{n})=\stackrel{\leftrightarrow}{d}(t): \stackrel{\leftrightarrow}{\varepsilon}_{\times}(\vec{n})
\end{aligned}
$$

(which are time-dependent because of the rotation of the detector tensor $\stackrel{\leftrightarrow}{d}$ ), we can decompose the beam pattern functions as

$$
\begin{aligned}
& F_{+}(t ; \vec{n}, \psi)=a(t ; \vec{n}) \cos 2 \psi+b(t ; \vec{n}) \sin 2 \psi, \\
& F_{\times}(t ; \vec{n}, \psi)=b(t ; \vec{n}) \cos 2 \psi-a(t ; \vec{n}) \sin 2 \psi
\end{aligned}
$$

The polarization angle is a property of the source, but the functions $a(t ; \vec{n})$ and $b(t ; \vec{n})$ depend on both the sky position of the source and the detector in question.

\section{Isolated neutron stars}

The relation between the detector time $t$ and the neutron star time $\tau$ depends on whether the neutron star is isolated or in a binary. For an isolated neutron star, we assume ${ }^{2}$ that the star is at rest with respect to the solar system barycenter (SSB) frame. Let $\vec{r}(t)$ be the position of the detector in the SSB frame and $\vec{v}(t)$ its velocity. The times of arrival of the wave at the detector and the SSB are

$$
t=\tau-\frac{\vec{r} \cdot \vec{n}}{c}+\text { relativistic corrections. }
$$

The relativistic corrections can be ignored for our purposes. The instantaneous frequency is then, to a very

\footnotetext{
${ }^{2}$ As it turns out, so long as the neutron star is moving inertially, this assumption is not necessary; the frequencies involved are all simply offset by the constant Doppler shift between the neutron star rest frame and the solar system barycenter.
} 
good approximation,

$$
\begin{gathered}
f(t)=\hat{f}(t)+\hat{f}(t) \frac{\vec{v} \cdot \vec{n}}{c}, \\
\hat{f}(t)=f_{0}+f_{1} t .
\end{gathered}
$$

The parameters of the signal from an isolated neutron star are thus the so-called amplitude parameters (or nuisance parameters) $\left\{h_{0}, \cos \iota, \psi, \Phi_{0}\right\}$ and the Doppler parameters $\boldsymbol{\lambda}=\left\{\vec{n}, f_{0}, f_{1}, \ldots\right\}$. The Doppler parameters determine the frequency evolution of the signal through (2.10). The frequency and spin-down ranges will canonically be taken to be $50 \mathrm{~Hz}<f_{0}<1000 \mathrm{~Hz}$, and $-1 \times 10^{-8} \mathrm{~Hz} / \mathrm{s}<f_{1}<$ 0 . These were the ranges used in [6]. The lowest frequency is determined by the performance of the detector, and it will be lower for the advanced detectors. The upper end of the frequency range could conceivably be as high as $2000 \mathrm{~Hz}$ depending on the computational cost.

Written in terms of the detector time $t$, and including first spin-downs, the phase is

$$
\Phi(t)=\Phi_{0}+2 \pi\left(f_{0} t+\frac{1}{2} f_{1} t^{2}\right)+2 \pi\left(f_{0}+f_{1} t\right) \frac{\vec{r} \cdot \vec{n}}{c} .
$$

We have ignored the $\frac{1}{2} f_{1}(\vec{r} \cdot \vec{n} / c)^{2}$ term. In fact, even the term $f_{1} t(\vec{r} \cdot \vec{n} / c)$ will be ignored in most of the calculations below. It is worth mentioning that all of these approximations are used only for our calculations in this paper, e.g. for estimating the search sensitivity and the parameter space resolution. On the other hand, for carrying out the actual search itself, it is important to know which frequency bins to correlate between different short data segments, and this in turn requires that we should be able to predict the frequency of a signal at any given time with sufficient accuracy. In particular, the relativistic Einstein and Shapiro corrections should of course not be ignored unless justified. The cross-correlation statistic described below in Sec. IV is general enough that as long as the frequency evolution is known sufficiently accurately, all the calculations go through unchanged.

Let us quantify the restrictions on the parameter space due to these approximations adapting the " $1 / 4$-cycle criterion" used in [24]: any physical effect which contributes less than $1 / 4$ of a cycle to the phase of the signal over a given coherent observation time will be ignored. Since $\mid \vec{r}$. $\vec{n} / c \mid \leq 1 \mathrm{AU} / c \approx 500 \mathrm{~s}$, we will have $\frac{1}{2}\left|f_{1}\right|(\vec{r} \cdot \vec{n} / c)^{2}<$ $1 / 4$ if $\left|f_{1}\right|<2 \times 10^{-6} \mathrm{~Hz} / \mathrm{s}$. This is much larger than any spin-downs we can realistically consider. On the other hand, the $f_{1} t(\vec{r} \cdot \vec{n} / c)$ is, in general, not negligible for realistic spin-downs and observation times of months. However, we will break up our observation time into shorter segments of duration much less than a day. Over say 1 hour, this term is ignorable if $\left|f_{1}\right|<3 \times 10^{-7} \mathrm{~Hz} / \mathrm{s}$ which is still a very large spin-down.

\section{Neutron stars in binary systems}

To account for the motion of the neutron star in a binary orbit, we need to add the orbital time delays to (2.10). The most important contribution is again the Roemer delay:

$$
t=\tau-\frac{\vec{r} \cdot \vec{n}}{c}+\frac{\vec{r}_{\text {orb }} \cdot \vec{n}}{c}+\text { relativistic corrections. }
$$

Here $\vec{r}_{\text {orb }}$ is the position vector of the neutron star in the binary system's center-of-mass frame.

There are four relevant orbital parameters. The first is the orbital period $P_{\text {orb }}$, and, if available, its derivative $\dot{P}_{\text {orb }}$. We then need a reference time within the orbit for which we use $T_{\text {asc }}$, the time of crossing of the ascending node. The third parameter is the projected semimajor axis of the neutron star, $a_{\mathrm{p}}=a_{\mathrm{x}} \sin i$. The final parameter is the orbital eccentricity $e$. In addition, there are 2 parameters specifying the orientation of the orbital plane, i.e. the inclination angle $i$ (not to be confused with the orientation of the neutron star axis $\iota$ ) and the argument of periapsis $\omega$. Of these 6 parameters, only 5 are required to define the phase model because of the projection along the line of sight $\vec{n}$; see [25] for further details.

We therefore have a total of 5 parameters of the binary which determine the frequency evolution of the signal: $\boldsymbol{\lambda}_{\mathrm{bin}}=\left(a_{\mathrm{x}} \sin i, e, P_{\mathrm{orb}}, T_{\mathrm{asc}}, \omega\right)$. In the case when the orbit is circular $(e=0)$, the argument of periapsis and the initial orbital phase combine additively into a single parameter so that we are left with only 3 search parameters: $\boldsymbol{\lambda}_{\text {bin }}=$ $\left(a_{\mathrm{p}}, P_{\text {orb }}, T_{\text {asc }}\right)$. We will not include higher derivatives of $P_{\text {orb }}$. As an example, for Sco X-1 (the brightest LMXB), some of the orbital parameters are $P_{\text {orb }} \approx 6.8 \times 10^{4} \mathrm{~s}$, and $a_{\mathrm{p}} / c \approx 1.44 \mathrm{~s}$, and $e<3 \times 10^{-3}[4,26,27]$.

Let $\vec{v}_{\text {orb }}$ be the velocity of the neutron star in the centerof-mass frame of the binary. The observed frequency is, to a very good approximation, given again by the nonrelativistic expression,

$$
f(t)=\hat{f}(t)+\hat{f}(t) \frac{\left(\vec{v}-\vec{v}_{\mathrm{orb}}\right) \cdot \vec{n}}{c} .
$$

Since $\vec{v}_{\text {orb }}$ is usually much larger than the Earth's orbital velocity, $\vec{v}_{\text {orb }}$ is the dominant contribution to the Doppler shift.

\section{B. Short-time Fourier transforms}

Given a time series detector output from a detector, it is convenient to break it up into short segments of length $\Delta T$ and to store the short-time Fourier transforms (SFTs). The value of $\Delta T$ is chosen such that the approximation (2.24) is valid and, as we will see, this leads to different restrictions on $\Delta T$ for neutron stars which are isolated or in binary systems. Such SFT databases are commonly used in the LIGO, GEO, and Virgo collaborations for periodic wave searches, and we will also base our data analysis strategies mostly on SFTs [28]. 
Let $x(t)$ be a time series sampled discretely at intervals of $\delta t$. Let us consider $N$ samples $x_{j}$ for $j=0 \ldots N-1$, and let $\Delta T=N \delta t$. Our convention for the discrete Fourier transform will be

$$
\tilde{x}_{k}=\delta t \sum_{j=0}^{N-1} x_{j} e^{-i 2 \pi j k / N},
$$

where $k=0,1 \ldots(N-1)$. For $0 \leq k \leq\lfloor N / 2\rfloor$, the frequency index $k$ corresponds to a physical frequency $f_{k}=$ $k / \Delta T$ with l.J denoting the integer part of a given real number. The values $\lfloor N / 2\rfloor<k \leq N-1$ correspond to negative frequencies given by $f_{k}=(k-N) / \Delta T$. Each SFT stores the real and imaginary values of $\tilde{x}_{k}$ for a range of frequency bin indices $k$. The $I$ th SFT will span the time interval $\left[T_{I}-\Delta T / 2, T_{I}+\Delta T / 2\right]$. When necessary, we will denote the data at the $k$ th frequency bin of the $I$ th SFT by $\tilde{x}_{k, I}$.

Equation (2.16) is actually a simplification. In practice, to avoid spectral leakage, a taper $w_{j}$ is applied while taking the Fourier transform:

$$
\tilde{x}_{k}=\delta t \sum_{j=0}^{N-1} w_{j} x_{j} e^{-i 2 \pi j k / N} .
$$

See e.g. [29] for details. We will mostly ignore windowrelated issues in this paper.

The detector output $x(t)$ is the sum of noise $n(t)$ plus a possible gravitational wave signal:

$$
x(t)=n(t)+h(t) .
$$

We will assume the noise to be a real stochastic process of zero mean, stationary and Gaussian; in practice, we only need stationarity over a period $\Delta T$, the time baseline of the SFTs. The properties of the noise are thus fully described by a single-sided power spectral density (PSD) $S_{n}(f)$ which, in the continuous time case is defined as

$$
S_{n}(f):=2 \int_{-\infty}^{\infty}\left\langle n\left(t^{\prime}+t\right) n\left(t^{\prime}\right)\right\rangle e^{-i 2 \pi f t} d t,
$$

where $\langle\cdot\rangle$ denotes an average over an ensemble of noise realizations. Note that the average $\left\langle n\left(t^{\prime}+t\right) n\left(t^{\prime}\right)\right\rangle$ is independent of $t^{\prime}$ because of the assumption of stationarity. In practice, we are of course only given $x(t)$ and not $n(t)$ itself. So we must take care to ensure that the estimation of $S_{n}(f)$ is not biased by the presence of a signal. Finally, the following expression for $S_{n}$ is useful:

$$
\left\langle\left|\tilde{x}_{k}\right|^{2}\right\rangle \approx \frac{\Delta T}{2} S_{n}\left(f_{k}\right) \text {. }
$$

This equation relates the variance of the (real and imaginary parts) of $\tilde{n}_{k}$ to the PSD, thus providing a more intuitive understanding of the PSD. This is a special case of a more general expression which, in the continuous case, reads

$$
\left\langle\tilde{n}^{*}(f) \tilde{n}\left(f^{\prime}\right)\right\rangle=\frac{1}{2} S_{n}(f) \delta\left(f-f^{\prime}\right) .
$$

\section{The short-duration Fourier transform of the signal}

We now calculate the Fourier transform of the signal over an observation duration $[T-\Delta T / 2, T+\Delta T / 2]$ centered at the time $T$. We assume $\Delta T$ is small enough so that $\left\{F_{A} \mid A=+, \times\right\}$ can be treated as constants in this duration; this means $\Delta T \ll 1$ day. We assume that the observation duration is small enough so that the phase of the signal in this duration can be expanded in a power series at the midpoint $T$ :

$$
\Phi(t)=\Phi(T)+2 \pi f(T)(t-T) .
$$

The validity of this approximation sets the limits on how large $\Delta T$ can be. If $\dot{f}(t)$ is the time derivative of the signal frequency at any given time $t$, the above approximation is valid whenever effects of the frequency derivative $\dot{f}$ can be ignored over the duration $\Delta T$. Using the $1 / 4$-cycle criterion, this leads to $\dot{f} \leq(\Delta T)^{-2}$.

For isolated neutron stars, the time variation of $f(t)$ is given by (2.11) and is due to two effects: the intrinsic spindown of the star, and the Doppler modulation due to the Earth's motion. Consider first the intrinsic spin-down $f_{1}$. Taking the largest spin-down to be $10^{-8} \mathrm{~Hz} / \mathrm{s}$, we get $\Delta T<10^{4}$ s. For the Doppler shift, we can estimate $\dot{f}$ by keeping $\hat{f}$ fixed and differentiating $\vec{v}$ in (2.11) or (2.15) as appropriate. The result for isolated neutron stars obeying (2.11) is worked out in [11] and yields the following restriction on $\Delta T$ :

$$
\Delta T<4 \times 10^{3} \mathrm{~s} \times \sqrt{\frac{500 \mathrm{~Hz}}{f_{0}}} .
$$

In this paper, for isolated neutron stars, we will mostly use $\Delta T=30 \mathrm{~min}$ as a canonical reference value. This is well within the above restrictions. The limits on $\Delta T$ are far more stringent for neutron stars in binary systems because of the higher Doppler shifts. The Sco X-1 search in [4] used $\Delta T=60 \mathrm{~s}$.

With the approximation (2.22), in the time interval $[T-$ $\Delta T / 2, T+\Delta T / 2]$, we have

$$
\begin{aligned}
h(t)= & F_{+} A_{+} \cos (\Phi(T)+2 \pi f(T)(t-T)) \\
& +F_{\times} A_{\times} \sin (\Phi(T)+2 \pi f(T)(t-T)) .
\end{aligned}
$$

The Fourier transform of $h(t)$ is easily seen to be

$$
\begin{aligned}
\tilde{h}(f)= & \int_{T-\Delta T / 2}^{T+\Delta T / 2} h(t) e^{-i 2 \pi f(t-T+\Delta T / 2)} d t \\
= & e^{i \pi f \Delta T}\left[e^{i \Phi(T)} \frac{\left(F_{+} A_{+}-i F_{\times} A_{\times}\right)}{2} \delta_{\Delta T}(f-f(T))\right. \\
& \left.+e^{-i \Phi(T)} \frac{\left(F_{+} A_{+}+i F_{\times} A_{\times}\right)}{2} \delta_{\Delta T}(f+f(T))\right],
\end{aligned}
$$


where we have defined the finite time approximation $\delta_{\Delta T}(f):=\sin (\pi f \Delta T) / \pi f$ to the delta function $\delta(f)$. This definition of the function $\delta_{\Delta T}(f)$ leads to significant spectral leakage of the signal power into neighboring frequency bins. This can be improved by using suitable tapers as in (2.17). We assume that this has been done and we will henceforth assume that spectral leakage is negligible.

\section{THE CROSS-CORRELATION STATISTIC FOR A PAIR OF SFTs}

Let us assume that we have two data streams covering the time intervals $I_{I}$ and $I_{J}$ centered on the times $T_{I}$ and $T_{J}$, respectively; both intervals have the same duration $\Delta T$. The data streams $x_{I}$ and $x_{J}$ in the two intervals could come from the same or different detectors, though of course if $T_{I}=T_{J}$ then the detectors have to be different. The received signals in the two intervals are denoted by $h_{I}(t)(t \in$ $\left.I_{I}\right)$ and $h_{J}(t)\left(t \in I_{J}\right)$, respectively. As before, we assume that the duration $\Delta T$ of the time intervals is such that the beam pattern functions are approximately constant. We denote the PSDs of the noise in the two intervals by $S_{n}^{(I)}(f)$ and $S_{n}^{(J)}(f)$, respectively.

The basic cross-correlation statistic corresponding to a filter function $Q$ is

$$
\mathcal{S}_{I J}=\int_{T_{I}-\Delta T / 2}^{T_{I}+\Delta T / 2} d t \int_{T_{J}-\Delta T / 2}^{T_{J}+\Delta T / 2} d t^{\prime} x_{I}(t) x_{J}\left(t^{\prime}\right) Q_{I J}\left(t, t^{\prime}\right)
$$

We would like to understand how the optimal $Q_{I J}$ can be chosen. The optimal choice depends in fact on the kind of signals we are looking for. The analysis presented in [19] describes the optimal choice of $Q$ for stochastic signals, and here we will tailor our discussion to the periodic signals described earlier.

To get some intuition on the nature of $\mathcal{S}_{I J}$, let us evaluate $\mathcal{S}_{I J}$ in the frequency domain assuming that $Q_{I J}$ is time invariant: $Q\left(t, t^{\prime}\right)=Q\left(t-t^{\prime}\right)$. Keep in mind however that this will not be the optimal solution, and a more detailed analysis will be presented later.

It is easy to evaluate (3.1) by writing $x_{I}(t)$ in terms of its Fourier transform. Along the way we approximate $\delta_{\Delta T}$ by the delta function, but we however should not take $Q_{I J}(\tau)$ to be a rapidly decreasing function of $\tau$ as in [19]. Since our signals have long-term-phase coherence, $Q_{I J}(\tau)$ will also turn out to be periodic. In any case, we still end up with the simple expression,

$$
\mathcal{S}_{I J}=\int_{-\infty}^{\infty} d f \tilde{x}_{I}^{*}(f) \tilde{x}_{J}(f) \tilde{Q}_{I J}(f) .
$$

The mean value of $\mathcal{S}_{I J}$ over an ensemble of noise realizations is

$$
\mu_{I J}:=\left\langle\mathcal{S}_{I J}\right\rangle=\int_{-\infty}^{\infty} d f \tilde{h}_{I}^{*}(f) \tilde{h}_{J}(f) \tilde{Q}_{I J}(f) .
$$

Here we have assumed that the noise has zero mean, and that $n_{I}$ and $n_{J}$ are uncorrelated. If we assume further that $h_{I} \ll n_{I}$ then the standard deviation is approximately

$$
\sigma_{I J}^{2}=\frac{\Delta T}{2} \int_{0}^{\infty} d f S_{n}^{(I)}(f) S_{n}^{(J)}(f)\left|\tilde{Q}_{I J}(f)\right|^{2} .
$$

Furthermore, it can also be shown under the same assumptions, that $\mathcal{S}_{I J}$ and $\mathcal{S}_{J K}$ are uncorrelated for $K \neq I$ :

$$
\left\langle\mathcal{S}_{I J} \mathcal{S}_{J K}\right\rangle=\delta_{I K} \sigma_{I J}^{2}
$$

Thus, the correlation pairs formed from all pairs of distinct SFTs are statistically independent. Note, however, that the same is not true for the third order moments; for example $\left\langle\mathcal{S}_{I J} \mathcal{S}_{J K} \mathcal{S}_{K I}\right\rangle \neq 0$ even when the small signal approximation is valid. This is however not a problem for us because we will never need to calculate the third and higher order correlations between the $\left\{\mathcal{S}_{I J}\right\}$.

Equation (3.3) clearly demonstrates that taking $Q_{I J}\left(t, t^{\prime}\right)$ to be time invariant is, in general, suboptimal for the data analysis problem at hand. The signal frequencies $f_{I}=$ $f\left(T_{I}\right)$ and $f_{J}=f\left(T_{J}\right)$ at the midpoints of the two intervals are given by (2.11) (for an isolated system) or (2.15) (for a binary systems). In general, $f_{I}$ and $f_{J}$ may be quite distinct from each other, especially if the intervals are far apart in time. Our assumptions on $\Delta T$ ensure that the signal power is concentrated mostly in a single SFT frequency bin. Thus, no matter what we choose for $\tilde{Q}_{I J}(f)$, the overlap between $\tilde{h}_{I}$ and $\tilde{h}_{J}$ might be quite small. This will lead to a small $\mu_{I J}$ and thus a small signal-to-noise ratio $\mu_{I J} / \sigma_{I J}$. The fix is obvious: we need to shift the frequencies while constructing the cross-correlation statistic. So, if we define $\delta f_{I J}=f_{J}-f_{I}$, then

$$
\mathcal{S}_{I J}=\int_{-\infty}^{\infty} d f \tilde{x}_{I}^{*}(f) \tilde{x}_{J}\left(f+\delta f_{I J}\right) \tilde{Q}_{I J}\left(f+\delta f_{I J} / 2\right) .
$$

In the time domain, this corresponds to the non-timeinvariant filter:

$$
Q_{I J}\left(t, t^{\prime}\right)=e^{-i \pi\left(\delta f_{I J}\right)\left(t+t^{\prime}\right)} Q_{I J}\left(t-t^{\prime}\right) .
$$

The mean $\mu_{I J}$ becomes

$$
\begin{aligned}
\mu_{I J} & :=\left\langle\mathcal{S}_{I J}\right\rangle \\
& =\int_{-\infty}^{\infty} d f \tilde{h}_{I}^{*}(f) \tilde{h}_{J}\left(f+\delta f_{I J}\right) \tilde{Q}_{I J}\left(f+\delta f_{I J} / 2\right),
\end{aligned}
$$

and the variance $\sigma_{I J}^{2}$ is unchanged.

An important quantity for us is the signal cross correlation $\tilde{h}_{I}^{*}(f) \tilde{h}_{J}\left(f+\delta f_{I J}\right)$. We extract the amplitude term $h_{0}^{2}$ and the delta functions to define for $f>0$,

$$
\tilde{h}_{I}^{*}(f) \tilde{h}_{J}\left(f+\delta f_{I J}\right)=h_{0}^{2} \tilde{G}_{I J} \delta_{\Delta T}^{2}\left(f-f_{I}\right) .
$$

The signal cross-correlation function $\tilde{G}_{I J}$ is an important quantity, much like the overlap reduction function for 
stochastic searches defined in [19] (though $\tilde{G}_{I J}$ is not exactly analogous to the overlap reduction function).

Apart from the frequency $f$ and $T_{I}, T_{J}, \tilde{G}_{I J}$ is a function of the signal parameters, i.e. the amplitude parameters $\left\{h_{0}, \iota, \psi, \Phi_{0}\right\}$, the Doppler parameters $\boldsymbol{\lambda}$, and possibly the binary parameters $\boldsymbol{\lambda}_{\text {bin }}$. To avoid clutter, we will often drop the dependence of $\tilde{G}_{I J}$ on the signal parameters and $T_{I}, T_{J}$, and just write $\tilde{G}_{I J}$.

Using (2.25) it is easy to calculate $\tilde{G}_{I J}$. For $f>0$, the dominant contribution is

$$
\begin{gathered}
\tilde{G}_{I J}=\frac{1}{4} e^{-i \Delta \Phi_{I J}}\left\{\left(F_{I+} F_{J+} \mathcal{A}_{+}^{2}+F_{I \times} F_{J \times} \mathcal{A}_{\times}^{2}\right)\right. \\
\left.-i\left(F_{I+} F_{J \times}-F_{I \times} F_{J+}\right) \mathcal{A}_{+} \mathcal{A}_{\times}\right\}, \\
\Delta \Phi_{I J}=\Phi_{I}\left(T_{I}\right)-\Phi_{J}\left(T_{J}\right) .
\end{gathered}
$$

Here we have added the subscript $I$ and $J$ to the phase $\Phi$ to emphasize that $\Phi$ is detector dependent. For isolated neutron stars, with the approximations explained in Sec. II A 1, this leads to

$$
\Delta \Phi_{I J}=2 \pi \sum_{k=0}^{s} \frac{f_{k}}{k !}\left(T_{I}^{k+1}-T_{J}^{k+1}\right)+2 \pi f_{0} \frac{\Delta \vec{r}_{I J} \cdot \vec{n}}{c} .
$$

We have used (2.13), ignored the $f_{1} t(\vec{r} \cdot \vec{n} / c)$ term, and defined $\Delta \vec{r}_{I J}:=\vec{r}\left(T_{I}\right)-\vec{r}\left(T_{J}\right)$. Recall from (2.3) that $\mathcal{A}_{+, \times}$are the same as $A_{+, \times}$but without the factor of $h_{0}$.

We can now also average over $\cos \iota$ using the following relations:

$$
\begin{aligned}
\left\langle\mathcal{A}_{+}^{2}\right\rangle_{\cos \iota}=\frac{7}{15}, \quad\left\langle\mathcal{A}_{\times}^{2}\right\rangle_{\cos \iota} & =\frac{1}{3},(3.13 \mathrm{a}) \\
\left\langle\mathcal{A}_{+} \mathcal{A}_{\times}\right\rangle_{\cos \iota} & =0 .(3.13 \mathrm{~b})
\end{aligned}
$$

The average of $\tilde{\mathcal{G}}_{I J}$ over cos $\iota$ is thus

$$
\left\langle\tilde{G}_{I J}\right\rangle_{\cos \iota}=\frac{1}{60} e^{-i \Delta \Phi_{I J}}\left(7 F_{I+} F_{J+}+5 F_{I \times} F_{J \times}\right) .
$$

We can easily perform another average over the polarization angle $\psi$ using (2.9a):

$$
\begin{aligned}
\left\langle F_{I+} F_{J+}\right\rangle_{\psi} & =\left\langle F_{I \times} F_{J \times}\right\rangle_{\psi}=\frac{1}{2}\left(a_{I} a_{J}+b_{I} b_{J}\right) \\
& =d_{I a b} P_{c d}^{\mathrm{TT} \vec{n} a b} d_{J}^{c d},
\end{aligned}
$$

where

$$
P_{c d}^{\mathrm{TT} \vec{n} a b}=\frac{1}{2} \sum_{A=+, \times} \varepsilon_{A}^{a b} \varepsilon_{A c d}=\frac{1}{2} \sum_{A=+, \times} e_{A}^{a b} e_{A c d}
$$

is a projection onto symmetric traceless tensors transverse to $\vec{n}$. This leads to

$$
\begin{aligned}
\left\langle\tilde{\mathcal{G}}_{I J}\right\rangle_{\cos \iota, \psi} & =\frac{1}{10} e^{-i \Delta \Phi_{I J}}\left(a_{I} a_{J}+b_{I} b_{J}\right) \\
& =\frac{1}{5} d_{I a b} d_{J}^{c d} P_{c d}^{\mathrm{TT} \vec{n} a b} e^{-i \Delta \Phi_{I J}} .
\end{aligned}
$$

In the case of time-coincident SFTs, since $\Delta \Phi_{I J}$ reduces to $\frac{\Delta \vec{r}_{I J} \cdot \vec{n}}{c}$, this is just a normalization factor times the overlap reduction function which would be used for a search for a stochastic background coming from a single point on the sky $[7,18,30,31]$.

\section{STATISTICS AND SENSITIVITY}

For each SFT pair (labeled by an index pair $I J$ ), we define the raw cross correlation as the complex random variable:

$$
\mathcal{Y}_{k, I J}=\frac{\tilde{x}_{k, I}^{*} \tilde{x}_{k^{\prime}, J}}{\Delta T^{2}}
$$

The frequency bin $k^{\prime}$ is shifted from $k$ by an amount corresponding to $\delta f_{I J}: k^{\prime}=k+\left\lfloor\Delta T \delta f_{I J}\right\rfloor$. Note that $\mathcal{Y}_{k, I J}$ is computed using only data from single frequency bins in the two SFTs; this works under the assumption that the signal power is mostly concentrated in a single frequency bin. We emphasize that this is not a fundamental limitation because we could, if we wished, consider the (optimally weighted) power from the neighboring bins as well if necessary. In the rest of this paper, we shall consider $\Delta T$ sufficiently small so that this assumption is valid. See Sec. II for quantitative estimates on $\Delta T$.

In this section we initially make two additional simplifying assumptions. First we take the signal to be much smaller than the noise, i.e. $h \ll n$, and second we only consider $\mathcal{Y}_{k, I J}$ for $I \neq J{ }^{3}$ The results obtained using these assumptions are probably the most relevant for practical applications. First, for ground-based detectors the signal is indeed expected to be much smaller than the noise. Second, the number of pairs of distinct SFTs is much more than the number of self pairs; there is thus no significant loss in sensitivity if the self-correlations are not considered in the final detection statistic.

The $\left\{\mathcal{Y}_{k, I J}\right\}$ are random variables with mean and variance given by

$$
\begin{gathered}
\mu_{k, I J}=h_{0}^{2} \tilde{\mathcal{G}}_{I J}, \\
\sigma_{k, I J}^{2}=\sigma_{k, I J}^{2}=\frac{1}{4 \Delta T^{2}} S_{n}^{(I)}\left(f_{k}\right) S_{n}^{(J)}\left(f_{k^{\prime}}\right) .
\end{gathered}
$$

To derive the expression for the mean, we have replaced $\delta_{\Delta T}\left(f-f_{I}\right)$ by $\delta_{\Delta T}(0)=\Delta T$, and for the variance we have assumed that the real and imaginary parts of $\tilde{x}_{k}$ are uncorrelated and have the same variance. The $\left\{\mathcal{Y}_{k, I J}\right\}$ are not Gaussian variables, but we will only need their mean and standard deviation.

Where convenient, we will replace the pair $I J$ with a single lowercase Greek index $\alpha, \beta \ldots$ Thus, $\mathcal{Y}_{k, I J}$ will often be denoted $\mathcal{Y}_{k, \alpha}$. To avoid unnecessary clutter, we also avoid putting the frequency index $k$ explicitly in $\mathcal{Y}_{k, \alpha}$.

\footnotetext{
${ }^{3}$ Both of these assumptions will be relaxed in the Appendix.
} 
In any case, one expects the signal contribution to be limited essentially to a single frequency bin $k$. Our task is now to combine the $\left\{\mathcal{Y}_{\alpha}\right\}$ in a statistically optimal way to extract the signal amplitude $h_{0}$. The following analysis is very similar to what is used in [6] (see also [10,32]).

We consider detection statistics which are weighted sums of the $\left\{\mathcal{Y}_{\alpha}\right\}$ :

$$
\rho=\sum_{\alpha}\left(u_{\alpha} \mathcal{Y}_{\alpha}+u_{\alpha}^{*} \mathcal{Y}_{\alpha}^{*}\right)
$$

We are interested in the probability distribution of the random variable $\rho$ because this is required for computing the sensitivity at given false alarm and false dismissal rates. It is simply obtained by examining the behavior of the noise in $\mathcal{Y}_{\alpha}$ (of which $\rho$ is made up of) which is derived from (4.1) by replacing the data $x$ by the noise $n$ in each data segment $I, J$. If we assume that the noise in each detector is Gaussian with mean zero, the noise in $\rho$ is a sum of products of real independent Gaussian variables each having mean zero. Although $\mathcal{Y}_{\alpha}$ is complex, the statistic $\rho$ is real. The product of two independent Gaussian variables whose mean is zero, is a random variable whose probability density function (PDF) is essentially $K_{0}(x)$, where $K_{0}(x)$ is the modified Bessel function of the second kind of order zero-more specifically, if $X \sim N\left(0, \sigma_{X}\right)$ and $Y \sim$ $N\left(0, \sigma_{Y}\right)$, then the PDF of $Z=X Y$ is $K_{0}\left(|z| / \sigma_{X} \sigma_{Y}\right) / \pi \sigma_{X} \sigma_{Y}$. This distribution has zero mean and a finite variance, namely, $\sigma_{X}^{2} \sigma_{Y}^{2}$. Then a generalization of the central limit theorem states that the sum of a large number of such zero mean variables tends to a Gaussian random variable [33]. Thus $\rho$ is a Gaussian random variable whose mean $\mu$ and variance $\sigma^{2}$ are given by

$$
\begin{gathered}
\mu=\sum_{\alpha}\left(u_{\alpha} \mu_{\alpha}+u_{\alpha}^{*} \mu_{\alpha}^{*}\right)=h_{0}^{2} \sum_{\alpha}\left(u_{\alpha} \tilde{G}_{\alpha}+u_{\alpha}^{*} \tilde{\mathcal{G}}_{\alpha}^{*}\right), \\
\sigma^{2}=2 \sum_{\alpha}\left|u_{\alpha}\right|^{2} \sigma_{\alpha}^{2} .
\end{gathered}
$$

Let us set a threshold $\rho_{\text {th }}$ on $\rho$ to select detection candidates based on a false alarm rate $\alpha$. It is easy to show that for Gaussian noise the threshold must be

$$
\rho_{\text {th }}=\sqrt{2} \sigma \operatorname{erfc}^{-1}(2 \alpha),
$$

where erfc is the complementary error function. The detection rate in the presence of a signal is

$$
\gamma=\frac{1}{2} \operatorname{erfc}\left(\frac{\rho_{\text {th }}-\mu}{\sqrt{2} \sigma}\right)
$$

Since $\mu \propto h_{0}^{2}$, this can be inverted to give the smallest value of $h_{0}$ that will cross the threshold at given false alarm and detection rates,

$$
h_{0}^{2}=2 \mathcal{S}\left(\frac{\sqrt{\sum_{\alpha}\left|u_{\alpha}\right|^{2} \sigma_{\alpha}^{2}}}{\sum_{\alpha}\left(u_{\alpha} \tilde{G}_{\alpha}+u_{\alpha}^{*} \tilde{G}_{\alpha}^{*}\right)}\right),
$$

where $\mathcal{S}=\operatorname{erfc}^{-1}(2 \alpha)-\operatorname{erfc}^{-1}(2 \gamma)$. This can also be written in terms of the false dismissal rate $\beta=1-\gamma$ as $\mathcal{S}=\operatorname{erfc}^{-1}(2 \alpha)+\operatorname{erfc}^{-1}(2 \beta){ }^{4}$ The solution for $u_{\alpha}$ which minimizes $h_{0}$ can then be shown to be

$$
u_{\alpha} \propto \frac{\tilde{G}_{\alpha}^{*}}{\sigma_{\alpha}^{2}} .
$$

It is shown in the Appendix that this solution also holds when we include the self-correlations (still assuming $h \ll$ $n)$.

Substituting from (4.10) into (4.4), the optimal detection statistic is

$$
\rho \propto \sum_{\alpha} \frac{\mathcal{Y}_{\alpha} \tilde{G}_{\alpha}^{*}+\mathcal{Y}_{\alpha}^{*} \tilde{G}_{\alpha}}{\sigma_{\alpha}^{2}}
$$

Substituting the expression for $u_{\alpha}$ from (4.10) back into (4.9), the optimal sensitivity is seen to be

$$
h_{0}=\left(\frac{\mathcal{S}^{2}}{\sum_{\alpha}\left|\tilde{G}_{\alpha}\right|^{2} / \sigma_{\alpha}^{2}}\right)^{1 / 4} \text {. }
$$

In the case when we are correlating data from two distinct interferometers with stationary noise floors $S_{n}^{(1)}(f)$ and $S_{n}^{(2)}(f)$, then $\sigma_{\alpha}$ is independent of $\alpha$ and is given by

$$
\sigma_{\alpha}^{2}=\frac{1}{4 \Delta T^{2}} S_{n}^{(1)}(f) S_{n}^{(2)}(f) .
$$

We are using the superscripts in $S_{n}^{(1)}$ and $S_{N}^{(2)}$ to refer to the two detectors. Similarly, if we denote the average of $\left|\tilde{G}_{\alpha}\right|^{2}$ over pairs of SFTs by $\left\langle\left|\tilde{G}_{\alpha}\right|^{2}\right\rangle_{\alpha}$, then

$$
\sum_{\alpha}\left|\tilde{G}_{\alpha}\right|^{2}=N_{\text {pairs }}\left\langle\left|\tilde{G}_{\alpha}\right|^{2}\right\rangle_{\alpha},
$$

where $N_{\text {pairs }}$ is the total number of SFT pairs. This leads to

$$
h_{0}=\frac{\mathcal{S}^{1 / 2}}{\sqrt{2}\left\langle\left|\tilde{G}_{\alpha}\right|^{2}\right\rangle_{\alpha}^{1 / 4}} \frac{1}{N_{\text {pairs }}^{1 / 4}} \sqrt{\frac{\left(S_{n}^{(1)} S_{n}^{(2)}\right)^{1 / 2}}{\Delta T}} .
$$

Similarly, if we relax the requirement that the pairs have to be from the distinct detectors, and instead assume that the noise floor in all SFTs is the same, $S_{n}$, then

$$
h_{0}=\frac{\mathcal{S}^{1 / 2}}{\sqrt{2}\left\langle\left|\tilde{G}_{\alpha}\right|^{2}\right\rangle_{\alpha}^{1 / 4}} \frac{1}{N_{\text {pairs }}^{1 / 4}} \sqrt{\frac{S_{n}}{\Delta T}} .
$$

These are the equations we were after. They give us the sensitivity of the cross-correlation search as a function of

\footnotetext{
${ }^{4}$ This is proved by using the following property of the complementary error function: $\operatorname{erfc}(-x)=2-\operatorname{erfc}(x)$. Setting $x=$ $-\operatorname{erfc}^{-1}(2 \gamma)$, we get $2-2 \beta=2 \gamma=\operatorname{erfc}(-x)=2-\operatorname{erfc}(x)$, which yields $x=\operatorname{erfc}^{-1}(2 \beta)$.

${ }^{5}$ This is perhaps easiest to see if we define a positive-definite inner product over vectors $\mathbf{x}=\left\{x_{\alpha}\right\}$ as $\mathbf{x} \cdot \mathbf{y}:=\sum_{\alpha} \operatorname{Re}\left[x_{\alpha}^{*} y_{\alpha}\right] \sigma_{\alpha}^{2}$. In terms of this inner product (4.9) can be written as $h_{0}=\mathcal{S} \frac{\|\mathbf{u}\|}{\mathbf{u} \cdot \mathbf{H}}$, where $H_{\alpha}=\tilde{G}_{\alpha}^{*} / \sigma_{\alpha}^{2} . h_{0}$ is then minimum when $\mathbf{u}$ is parallel to H.
} 
the statistical false alarm and false dismissal rates, the SFT baseline $\Delta T$, the noise floors of the SFTs, the number of SFT pairs $N_{\text {pairs }}$, and the geometrical factors contained in $\tilde{G}_{\alpha}$. They tell us that the sensitivity grows coherently with $\Delta T$ and incoherently with $N_{\text {pairs }}$. Note however that we can correlate any SFT pair we like, so that $N_{\text {pairs }}$ can be made much larger than the number of SFTs $N_{\text {sft }}$ (even if we were to exclude self-correlations). In fact, if we believe the signal to maintain phase coherence over the entire observation time (which may be months or years), and if we can afford to do so computationally, then $N_{\text {pairs }} \sim N_{\text {sft }}^{2}$ so that $h_{0} \propto\left(N_{\text {sft }} \Delta T\right)^{-1 / 2}$ which is better than what we would get with the standard semicoherent searches [6].

\section{THE RELATION WITH THE $\mathcal{F}$ STATISTIC}

From (4.15), we see that if we use all SFT pairs available, the amplitude sensitivity of the cross-correlation search is proportional to $T_{\mathrm{obs}}^{-1 / 2}$ which is what we would get for a fully coherent search. There must thus be a close relation between the cross correlation and the coherent matched filter, and in this section we show that this is indeed the case.

A convenient implementation of the matched filter statistic for periodic waves is provided by the so-called $\mathcal{F}$-statistic first defined in [24] for the single interferometer case, and later generalized to the multi-interferometer case in [17], and a detailed study of the parameter space resolution was presented in [34]. Let us start with the single interferometer case.

For defining the $\mathcal{F}$-statistic, it is convenient to rewrite the waveform of (2.2). We first separate out the initial phase $\Phi_{0}$ from the total phase as

$$
\Phi(t)=\Phi_{0}+\varphi(t) .
$$

We decompose the total waveform $h(t)$ in terms of four quadratures as

$$
h(t)=\sum_{i=1}^{4} \mathcal{A}^{\mu} h_{\mu}(t)
$$

where the four amplitudes $\left\{\mathcal{A}^{\mu}\right\}$ (not to be confused with $\mathcal{A}_{+}$and $\mathcal{A}_{\times}$) are time independent and the $\left\{h_{\mu}\right\}$ are

$$
\begin{aligned}
h_{1}(t) & =a(t) \cos \varphi(t), & & h_{2}(t)=b(t) \cos \varphi(t), \\
h_{3}(t) & =a(t) \sin \varphi(t), & & h_{4}(t)=b(t) \sin \varphi(t),
\end{aligned}
$$

with $a(t)$ and $b(t)$ defined as in (2.8). What this decomposition achieves is a separation of the amplitude parameters $\left\{h_{0}, \iota, \psi, \Phi_{0}\right\}$ from the Doppler parameters. The only signal parameters in the quadratures $\left\{h_{\mu}\right\}$ are the Doppler parameters while the amplitudes $\left\{\mathcal{A}^{\mu}\right\}$ depend only on the amplitude parameters.

In order to extract the signal $h(t)$ from the noise, the optimal search statistic is the likelihood function $\Lambda$ defined by

$$
\ln \Lambda=(x \mid h)-\frac{1}{2}(h \mid h),
$$

where the inner product $(\cdot \mid \cdot)$ is defined as

$$
(x \mid y):=2 \int_{0}^{\infty} \frac{\tilde{x}(f) \tilde{y}^{*}(f)+\tilde{x}^{*}(f) \tilde{y}(f)}{S_{n}(f)} d f .
$$

The quantity $\ln \Lambda$ is essentially the matched filter and is precisely what we should use in order to best detect the waveform $h(t)$. An explicit search over the amplitude parameters $\left\{\mathcal{A}^{\mu}\right\}$ is avoided by noting that $\ln \Lambda$ depends quadratically on the $\left\{\mathcal{A}^{\mu}\right\}$. We can thus analytically find the maximum likelihood (ML) estimators $\left\{\hat{\mathcal{A}}^{\mu}\right\}$ of the amplitudes $\left\{\mathcal{A}^{\mu}\right\}$ by solving the set of four coupled linear equations:

$$
\left.\frac{\partial \ln \Lambda}{\partial \mathcal{A}^{\mu}}\right|_{\mathcal{A}^{\nu}=\hat{\mathcal{A}}^{\nu}}=0, \quad \mu=1, \ldots, 4 .
$$

The $\mathcal{F}$-statistic is then defined as the log likelihood ratio with the values of the amplitudes $\left\{\mathcal{A}^{\mu}\right\}$ replaced by their ML estimators:

$$
\mathcal{F}:=\left.\ln \Lambda\right|_{\mathcal{A}^{\mu}=\hat{\mathcal{A}}^{\mu}} .
$$

Explicitly, $\mathcal{F}$ can be written as

$$
\mathcal{F}=\frac{4}{S_{n}\left(f_{0}\right)} \frac{B\left|F_{a}\right|^{2}+A\left|F_{b}\right|^{2}-C\left(F_{a} F_{b}^{*}+F_{b} F_{a}^{*}\right)}{A B-C^{2}},
$$

where

$$
\begin{aligned}
F_{a} & =\int_{0}^{T_{\mathrm{obs}}} x(t) a(t) e^{-i \varphi(t)} d t, \\
F_{b} & =\int_{0}^{T_{\mathrm{obs}}} x(t) b(t) e^{-i \varphi(t)} d t, \\
A & =\int_{0}^{T_{\mathrm{obs}}} a^{2}(t) d t, \quad B=\int_{0}^{T_{\mathrm{obs}}} b^{2}(t) d t, \\
C & =\int_{0}^{T_{\mathrm{obs}}} a(t) b(t) d t .
\end{aligned}
$$

We need to write the $F_{a}$ and $F_{b}$ still more explicitly; let us start with $F_{a}$. We break up the integral for $F_{a}$ into subintervals defined by the SFTs, and assume as we have been doing all along that $a(t)$ is constant over the SFT duration:

$$
\begin{aligned}
F_{a} & =\sum_{I} \int_{T_{I}+\Delta T / 2}^{T_{I}-\Delta T / 2} x(t) a(t) e^{-i \varphi(t)} d t \\
& =\sum_{I} a_{I} \int_{T_{I}+\Delta T / 2}^{T_{I}-\Delta T / 2} x(t) e^{-i \varphi(t)} d t .
\end{aligned}
$$

Writing the phase in a Taylor series around the SFT midtime and keeping the linear terms, we get

$$
\varphi(t)=\varphi\left(T_{I}\right)+i 2 \pi f_{I}\left(t-T_{I}\right)
$$

which leads to

$$
\begin{aligned}
F_{a} & =\sum_{I} a_{I} e^{-i \varphi\left(T_{I}\right)} \int_{T_{I}-\Delta T / 2}^{T_{I}+\Delta T / 2} x(t) e^{-i 2 \pi f_{I}\left(t-T_{I}\right)} d t \\
& =\sum_{I} a_{I} e^{-i \varphi\left(T_{I}\right)} e^{-i \pi f \Delta T} \tilde{x}_{I}\left(f_{I}\right),
\end{aligned}
$$

and likewise for $F_{b}$. 
Now we are ready to look at $\mathcal{F}$ again. From (5.8) it is clear that $\mathcal{F}$ is quadratic in the data and from (5.12) it is clear that we will end up with an expansion like

$$
\mathcal{F}=\sum_{I J} \mathcal{F}_{I J}
$$

In fact, it turns out that (5.13) is precisely a linear combination of the $\mathcal{Y}_{\alpha}$ defined in (4.1). Explicitly, it follows from (5.12) that

$$
\left|F_{a}\right|^{2}=\sum_{I J} a_{I} a_{J}\left(e^{i \Delta \Phi_{I J}} \mathcal{Y}_{I J}+e^{-i \Delta \Phi_{I J}} \mathcal{Y}_{I J}^{*}\right)
$$

Similar expressions are obtained for $\left|F_{b}\right|^{2}$ and the cross term $F_{a} F_{b}^{*}+F_{b} F_{a}^{*}$ of the $\mathcal{F}$ statistic. Combining all of the results from above, we see that $\mathcal{F}$ is a detection statistic of the form (4.4) with weights,

$$
u_{I J} \propto\left(A b_{I} b_{J}+B a_{I} a_{J}-C\left(a_{I} b_{J}+a_{J} b_{I}\right)\right) e^{i \Delta \Phi_{I J}} .
$$

In the case where $A \approx B$ and $C \ll A, B$, this is seen to be proportional to $\tilde{G}_{I J}$ averaged over $\cos \iota$ and $\psi$ (3.17). Thus we see that the cross-correlation statistic $\rho$ is indeed roughly equivalent to the $\mathcal{F}$-statistic. In principle, $\rho$ using the full signal cross-correlation function $\tilde{G}_{\alpha}$ from (3.10), is a function of the Doppler parameters and also of $\left\{A_{+}, A_{\times}, \psi\right\}$; this is more like the likelihood ratio (modulo the dependence on the initial phase $\Phi_{0}$ ) before maximizing it over the amplitude parameters to obtain the $\mathcal{F}$-statistic. The $\rho$ calculated with $\left\langle\tilde{G}_{\alpha}\right\rangle_{\cos \iota, \psi}$ is closer to the matched filter statistic marginalized over $\cos \iota$ and $\psi$.

\section{ESTIMATING THE AMPLITUDE PARAMETERS}

Thus far, we have focused on constructing the crosscorrelation statistic which is optimal for the detecting the presence of periodic GWs. Thus, the choice of weights given in (4.10) is tailored for measurements of excess cross-correlation power, and is not actually an estimator for the signal amplitude. Estimating the Doppler parameters $\left\{f_{0}, f_{1}, \ldots, \vec{n}\right\}$ is easy since we are searching over these parameters explicitly. Note also that the signal crosscorrelation function $\tilde{G}_{\alpha}$ of (3.10) is a function of cos $\iota$ and $\psi$. We could thus, in principle, find the values of $\cos \iota$ and $\psi$ which maximize $\rho$, thus yielding estimators of these quantities. In practice, however, we expect it to be more convenient to use a single statistic, such as that associated with the averaged $\tilde{G}_{\alpha}$ given in (3.17), and then estimate $\left\{A_{+}, A_{\times}, \psi\right\}$ in a follow-up stage. ${ }^{6}$ In this section, we show that it is indeed possible to estimate $\left\{A_{+}, A_{\times}, \psi\right\}$. The method presented here is a straightforward generalization of [35] (see also [6,10]) developed for the standard semicoherent searches.

\footnotetext{
${ }^{6}$ Note that the cross correlations $\mathcal{Y}_{\alpha}$ are independent of the initial phase $\Phi_{0}$. Thus, it is not possible to estimate $\Phi_{0}$ if we restrict ourselves to measurements of $\mathcal{Y}_{\alpha}$.
}

The basic idea is to note that the two polarizations $h_{+}$ and $h_{\times}$appear in the detector with different amplitude modulations. Therefore, given sufficient measurements of the $\mathcal{Y}_{\alpha}$, it should be possible to extract the signal components with different amplitude modulation patterns thereby estimating the amplitudes $A_{+}$and $A_{\times}$. Let us start by defining the signal cross-correlation functions $\tilde{\mathcal{G}}_{\alpha}^{+}$and $\tilde{\mathcal{G}}_{\alpha}^{\times}$for the two polarizations which are analogous to $\tilde{\mathcal{G}}_{\alpha}$ :

$$
\begin{aligned}
& \tilde{\mathcal{G}}_{I J}^{+}=\frac{1}{4} e^{-i \Delta \Phi_{I J}} F_{I+} F_{J+}, \\
& \tilde{G}_{I J}^{\times}=\frac{1}{4} e^{-i \Delta \Phi_{I J}} F_{I \times} F_{J \times} .
\end{aligned}
$$

These functions are significant because, just as in (4.2), they tell us about the mean $\mu_{\alpha}$ of $\mathcal{Y}_{\alpha}$ for the two independent polarizations. The contributions of $A_{+}$and $A_{\times}$to the mean are respectively

$$
\mu_{\alpha}^{+}=A_{+}^{2} \tilde{\mathcal{G}}_{\alpha}^{+} \quad \text { and } \quad \mu_{\alpha}^{\times}=A_{\times}^{2} \tilde{\mathcal{G}}_{\alpha}^{\times} .
$$

An estimator of $A_{+}$is obtained by minimizing the following $\chi^{2}$-statistic as a function of $A_{+}^{2}$ :

$$
\chi^{2}=\sum_{\alpha} \frac{\left|\mathcal{Y}_{\alpha}-A_{+}^{2} \tilde{G}_{\alpha}^{+}\right|^{2}}{\sigma_{\alpha}^{2}}
$$

The solution of $\partial \chi^{2} / \partial A_{+}^{2}=0$ is easily seen to be

$$
A_{+}^{2}=\left(\sum_{\beta} \frac{2\left|\tilde{\mathcal{G}}_{\beta}^{+}\right|^{2}}{\sigma_{\beta}^{2}}\right)^{-1} \sum_{\alpha} \frac{\mathcal{Y}_{\alpha}^{*} \tilde{\mathcal{G}}_{\alpha}^{+}+\mathcal{Y}_{\alpha} \tilde{\mathcal{G}}_{\alpha}^{+*}}{\sigma_{\alpha}^{2}}
$$

Similarly, the estimator for $A_{\times}$is

$$
A_{\times}^{2}=\left(\sum_{\beta} \frac{2\left|\tilde{G}_{\beta}^{\times}\right|^{2}}{\sigma_{\beta}^{2}}\right)^{-1} \sum_{\alpha} \frac{\mathcal{Y}_{\alpha}^{*} \tilde{\mathcal{G}}_{\alpha}^{\times}+\mathcal{Y}_{\alpha} \tilde{\mathcal{G}}_{\alpha}^{\times *}}{\sigma_{\alpha}^{2}}
$$

Since $\left\{\tilde{\boldsymbol{G}}_{\alpha}^{A}\right\}$ depend on the polarization angle $\psi$ through the beam pattern functions, both (6.4) and (6.5) imply a search over $\psi$. We expect these estimators to be better than the ones used in the standard semicoherent methods simply because it uses a larger number of measurements including all possible pairs of SFTs. Note that these estimators for $A_{+}^{2}$ and $A_{\times}^{2}$ are proportional to the optimal excess-power statistic $\rho$ of (4.11), with $\tilde{\mathcal{G}}_{\alpha}$ replaced by $\tilde{G}_{\alpha}^{+}$and $\tilde{\mathcal{G}}_{\alpha}^{\times}$.

Finally, while we do not discuss it here, following [35], this discussion can be generalized to construct a joint $\chi^{2}$ statistic for $A_{+}^{2}, A_{\times}^{2}$, and $\psi$ for a general elliptically polarized signal.

\section{PARAMETER SPACE RESOLUTION}

In this section we discuss the parameter space resolution required for the cross-correlation statistic $\rho$. This affects the astrophysical significance of the search in terms of parameter estimation and also the computational requirements for carrying out the search. The parameter space resolution for a detection statistic $\rho$ is usually discussed in 
terms of the parameter space metric. This is defined as the fractional loss in the signal-to-noise ratio when $\rho$ is calculated at a point in parameter space which is slightly different from the point corresponding to the actual signal parameters [36-38]. In our case, we are in principle free to consider any subset of all the possible SFT pairs in calculating the final detection statistic $\rho$. However, without some control on which SFT pairs are chosen, it seems very hard to get a handle on the parameter space metric for the general cross-correlation statistic $\rho$ defined by (4.4). Our suggestion is the following: Choose a time duration $T_{\max }$ and include only those SFT pairs $\{I, J\}$ for which $\mid T_{I}-$ $T_{J} \mid \leq T_{\max }$. Thus, $T_{\max }$ can be viewed as the maximum duration over which we choose to maintain strict phase coherence.

If $T_{\max }=T_{\mathrm{obs}}$ then we are including all possible pairs, and at the other extreme, if $T_{\max }=0$ then we are including only self-correlations and time-coincident correlations between different detectors. In the intermediate regime the cross-correlation search is closest in spirit to a semicoherent hierarchical scheme which consists of breaking up the total data available (say from $t=0$ to $t=T_{\mathrm{obs}}$ ) into shorter segments $\left[0, T_{\max }\right],\left[T_{\max }, 2 T_{\max }\right] \ldots$ One then performs a coherent analysis on each of the segments (using, say, the $\mathcal{F}$-statistic) and combines the results semicoherently $[9,11,12]$. The pair selection criteria would lead us to choose all possible SFT pairs within each of the segments. Since we have already seen in Sec. V that this is essentially equivalent to the $\mathcal{F}$-statistic, the similarities between the two schemes is obvious. The two schemes are however not exactly identical because this SFT pair selection criteria also includes choosing pairs lying in adjacent data segments (assuming the segments are sufficiently close to each other). Thus, the cross-correlation search with coherence time $T_{\max }$ will be more sensitive than the semicoherent search with coherent segments of duration $T_{\max }$ but the precise improvement depends on the duty cycle of the detectors, i.e. on the gaps between the SFTs and the coherent segments.

With this criteria of choosing pairs, we will see that the resolution depends on $T_{\max }$ the SFT baseline $\Delta T$. To make our results concrete, we will focus on ground-based interferometers by taking the frequency range to be from 50 to $1000 \mathrm{~Hz}$. Given the similarities with the semicoherent and hierarchical schemes discussed above, it is clear that a proper discussion of the metric requires a calculation of the parameter space metric for semicoherent searches. This is a combination of the coherent metric worked out in detail in [34,39], and the semicoherent metric obtained by summing $\mathcal{F}$-statistic segments. Preliminary calculations have been worked out in [9], but a detailed study of its properties is still lacking. We will instead resort to order of magnitude estimates (which, in spite of their approximate nature, have actually turned out to be fairly useful for previous searches; see e.g. [11]).
We can either use the amplitude modulation of the detection statistic $\rho=\sum_{\alpha} \rho_{\alpha}$ by which we mean the variation of $\rho_{\alpha}$ with $\alpha$, or we can use the frequency modulation reflected in the different frequency bins $k$ and $k^{\prime}$ used to calculate the cross correlation $\mathcal{Y}_{\alpha}=\tilde{x}_{k, I}^{*} \tilde{x}_{k^{\prime}, J}$. Starting with the sky resolution, we identify three factors which could be relevant: the detector beam pattern functions, the detectorpair baseline $\Delta \vec{r}_{I J}$, and the Doppler information over a duration $\Delta T$ and $T_{\max }$; we discuss all of these in turn. The relative importance of these three factors depends on the search parameters.

(i) The expectation value of the cross-correlation statistic varies with the SFT pair index $\alpha$, and part of this variation is due to the geometrical factor $a_{I} a_{J}+$ $b_{I} b_{J}$ in (3.11). Since this variation depends on the sky position, it can in principle be used to get skyposition information. The resolution thus obtained is roughly given by the angular scales over which the beam pattern functions vary. Note that this amplitude modulation is due to the rotation of Earth around its axis; this is independent of the signal frequency and gets mostly averaged out if $\Delta T$ is comparable or larger than a day.

(ii) The other reason for the variation of the signal-tonoise ratio with $\alpha$ is the $\Delta \vec{r}_{I J}$ term in (3.11). In the case when the two SFTs are coincident in time $\left(T_{I}=\right.$ $T_{J}$ ), then $\Delta \vec{r}_{I J}$ is the separation between the two detectors; for the LIGO Hanford and Livingston observatories, this corresponds to a light travel time of about $10 \mathrm{~ms}$. More generally, the magnitude of $\Delta \vec{r}_{I J}$ is the distance between the positions of the two (distinct or same) detectors at different times; it could be as much as $2 \mathrm{AU}$ if $T_{I}-T_{J} \sim 6$ months. On the other extreme, it could be zero if we are correlating the data with itself (which is what the standard semicoherent methods do); this effect then becomes completely irrelevant. If $\lambda_{\mathrm{gw}}$ is the wavelength of the wave we are trying to detect, the sky resolution associated with $\Delta \vec{r}_{I J}$ is inversely proportional to the frequency:

$$
(\Delta \theta)_{\Delta \vec{r}} \approx \frac{\lambda_{\mathrm{gw}}}{|\Delta \vec{r}|}=\frac{1}{f \cdot|\Delta \vec{r}| / c} .
$$

For the Hanford-Livingston pair, this corresponds to about $\mathcal{O}\left(60^{\circ}\right)$ at $100 \mathrm{~Hz}$ and about $6^{\circ}$ at $1000 \mathrm{~Hz}$.

(iii) The third way of getting sky-position information is through the Doppler shift. This is only useful if the frequency resolution of the SFTs is small enough; the maximum Doppler shift is $f|\vec{v}| / c$, so for the Doppler shift to be important, we must have

$$
\Delta T>\frac{\lambda_{\mathrm{gw}}}{|\vec{v}|} .
$$

The magnitude of Earth's orbital velocity in its orbit is $\sim 10^{-4} c$, so (7.2) leads to $\Delta T>200 \mathrm{~s}$ at $50 \mathrm{~Hz}$ and 
$\Delta T>6.67 \mathrm{~s}$ at $1500 \mathrm{~Hz}$. One relevant baseline in this case is the distance traveled by the detector in the duration $\Delta T$. Thus, the sky resolution is (see [11] for further details)

$$
(\Delta \theta)_{\text {Doppler }}=\frac{\lambda_{\mathrm{gw}}}{|\vec{v}| \Delta T} .
$$

For $1800 \mathrm{~s}$ SFTs, this corresponds to $\sim 6^{\circ}$ at $50 \mathrm{~Hz}$ and $0.2^{\circ}$ at $1500 \mathrm{~Hz}$. There is finally the baseline corresponding to $T_{\max }$, i.e. the distance $d_{\max }$ traveled by the detector during $T_{\max }$. This leads to

$$
(\Delta \theta)_{\text {Doppler }}=\frac{\lambda_{\mathrm{gw}}}{d_{\max }} .
$$

More generally, the resolution corresponding to $T_{\max }$ (for sufficiently large $\Delta T$ ) is precisely the coherent metric calculated in [34,39].

We see that the first two items above can be viewed as using the amplitude modulation information (dependence of the signal-to-noise ratio on the pair index $\alpha$ ), while the third uses the frequency modulation.

Let us now discuss the resolution in spin-down parameters $f_{k}$. The spin-down term in $\Delta \Phi_{I J}$ appears in the combination $f_{k}\left(T_{I}^{k+1}-T_{J}^{k+1}\right)$. Thus, it is clear that for $T_{I} \neq T_{J}$ this leads to a spin-down resolution of

$$
\left(\delta f_{k}\right)_{\min }=\frac{1}{\max _{I, J}\left\{\left|T_{I}^{k+1}-T_{J}^{k+1}\right|\right\}} .
$$

Thus, if we were to consider all possible pairs from a given set of SFTs, and if we define the reference time to be in the midpoint of the observation duration, then we would have $\delta f_{k} \propto T_{\mathrm{obs}}^{-(k+1)}$.
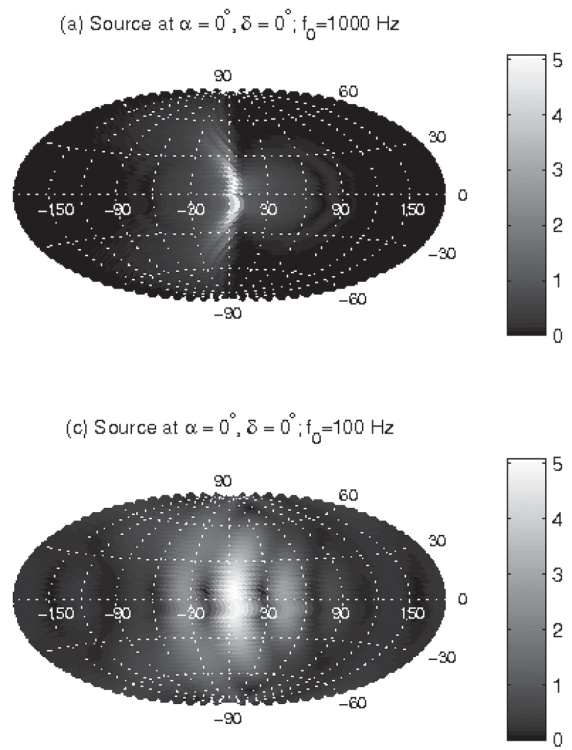

We can also consider the frequency resolution $(\delta f)_{\mathrm{sft}}=$ $(\Delta T)^{-1}$ of the SFTs themselves. The corresponding resolution in $f_{k}$ is defined by the smallest change in $f_{k}$ required to change the frequency by a $(\delta f)_{\text {sft }}$ over the full observation time $T_{\mathrm{obs}}$. This leads to $\delta f_{k}=(\delta f)_{\mathrm{sft}} / T_{\mathrm{obs}}^{k}$ for $k=$ $1,2 \ldots$

Let us conclude this section by giving a short numerical example for the case when we correlate data from a pair of spatially separated detectors at the same times. We consider frequencies of 100 and $1000 \mathrm{~Hz}$, and two sky positions: one at the celestial equator and one at $45^{\circ}$ degrees above it. In each case we consider sources with the optimal orientation $\iota=0$, without any spin-down parameters, and with $\psi=0$. The total observation time is taken to be $T_{\mathrm{obs}}=1 \mathrm{yr}$ and the SFT baseline is $\Delta T=30 \mathrm{~min}$. We assume that the two data streams are coming from the LIGO Livingston and Hanford interferometers. For performing the cross correlations, we use

$$
Q(t ; f, \vec{n})=\lambda(t ; \vec{n})\langle\tilde{G}(\vec{n})\rangle_{\cos \iota, \psi},
$$

where, $\lambda(t ; \vec{n})$ is a proportionality constant. We consider essentially identical time segments - same barycentric time - in the two detectors. In a year's worth of observation time there are little over 17000 such time segments, each of 30 minutes duration. Thus the time-segment indices $I, J$ each, sequentially run over the full observation time. The relevant quantities $Q, \tilde{G}$, and $\lambda$ in (7.6) which carry the same indices also do the same over the observation time - thus we may think of each of them as functions of $t$ - the segment time stamp; thus $I$ or $J$ is replaced by $t$.

For the signal only case, the cross correlation can be written explicitly as
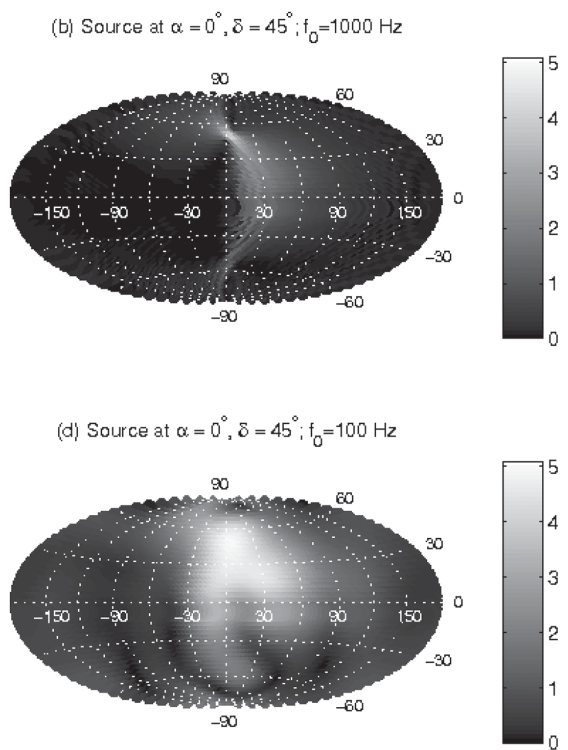

FIG. 1. The point spread functions (PSFs) for sources with frequencies $100 \mathrm{~Hz}$ [(c) and (d)] and $1000 \mathrm{~Hz}$ [(a) and (b)]. The source is taken at the celestial equator $\left[(a)\right.$ and (c)] and $45^{\circ}$ above the celestial equator [(b) and (d)]. In all the cases, the source orientation is taken to be optimal, i.e., $\iota=\psi=0$. 


$$
B\left(\vec{n}, \vec{n}^{\prime}\right)=\Lambda(\vec{n}) \int_{0}^{T_{\mathrm{obs}}}\langle\tilde{G}(t ; \vec{n})\rangle_{\cos \iota, \psi} h_{(1)}\left(t ; \vec{n}^{\prime}\right) h_{(2)}\left(t ; \vec{n}^{\prime}\right) d t,
$$

where the subscripts in $h_{(1)}$ and $h_{(2)}$ refer to the two distinct detectors we are considering. We have chosen

$$
\Lambda^{-1}(\vec{n})=\frac{1}{\Delta T} \int \lambda^{-1}(t ; \vec{n}) d t .
$$

We choose the proportionality constant $\lambda$ such that it is inversely proportional to the square of the average total power accessible to the network for a particular direction of the sky in the interval $\Delta T$ of the SFTs. Thus we have

$$
\lambda^{-1}(t ; \vec{n})=\Delta T\langle\tilde{G}(t ; \vec{n})\rangle_{\cos \iota, \psi}^{2} .
$$

This is in the spirit of the normalization scheme adopted in [20]. Figure 1 shows $B\left(\vec{n}, \vec{n}^{\prime}\right)$ evaluated numerically for point sources at different positions. We note that the maximum value of $B$ is 5 . This is the result of the average value of $\tilde{\mathcal{G}}$ we have chosen in defining the filter function together with the fact that we have chosen optimally oriented sources for the numerical computation. The sky resolution is characterized quantitatively by the FWHM (full width at half maximum) of the PSF. From the figures it turns out to be $\simeq 8^{\circ}$ for $f_{0}=1000 \mathrm{~Hz}$ and $\simeq 80^{\circ}$ for $f_{0}=100 \mathrm{~Hz}$. We observe that the agreement between the order of magnitude estimates obtained earlier and the actual values computed from the figure is satisfactory.

\section{DISCUSSION}

Let us summarize the main results of this paper. We have generalized the cross-correlation statistic, traditionally used for the stochastic gravitational wave background searches, to periodic gravitational waves. The features of periodic waves, not present in the stochastic background signals, are nonstationarity and long-term coherence. The nonstationarity may need to be taken into account depending on the frequency resolution, and the long-term coherence implies that we can in principle cross correlate data segments from arbitrary times and arbitrary detectors. In this framework, we can naturally consider a network consisting of an arbitrary number of detectors. Because of the freedom in choosing which data-segment pairs to correlate, the method is very flexible, and these are some of the possibilities:

(i) We can, if we wish, correlate all possible short data segments. If this is done, then we showed that the resulting detection statistic is very close to the $\mathcal{F}$-statistic corresponding to a full matched filter statistic. In this case the parameter space resolution becomes very fine and consequently, while this is ideally the most sensitive method, its computational cost becomes prohibitive for wide parameter space searches.

(ii) At the other extreme, we can choose to correlate only data segments taken from distinct detectors at the same (or very close) times. This is the closest in spirit to the standard directed stochastic background searches using aperture synthesis. In this mode of operation, the search is not computationally intensive, and is very robust against signal uncertainties. However, this also implies poor resolution in parameter space, and thus more expensive follow-ups to verify possible detections and to estimate the signal parameters.

(iii) From the perspective of this paper, the standard semicoherent searches such as PowerFlux, StackSlide, and Hough all correspond to the special case in which we consider only self-correlations [5,6,9-11]. The procedure of considering weighted sums of the cross-correlation power is closest to the PowerFlux method. In fact, many of the lessons learned in the PowerFlux searches should be applicable here with suitable modifications. For example, the estimation of the signal amplitudes developed originally for PowerFlux carries over rather straightforwardly.

(iv) In intermediate regimes when we correlate data segments separated by a maximum coherence time $T_{\max }<T_{\mathrm{obs}}$, the cross-correlation search is similar to a hierarchical search in which we combine segments of demodulated data. Though, as discussed in Sec. VII, there are differences between the two with the cross-correlation search being somewhat more sensitive.

Conceptually, this method thus provides a unified framework for all the known periodic wave searches, and this might be useful in various calculations and applications. Each of the above modes of operation correspond to tuning the maximum coherence time all the way from small values to the total observation time. The precise value chosen for a specific application depends on the tradeoffs between computational cost, sensitivity, and robustness against signal uncertainties. The additional parameter which figures importantly in this trade-off is the length $\Delta T$ of the short data segments. It is worth mentioning that there exist other analyses which consider the angular resolution of general multidetector arrays (e.g. [40]); these methods and results might be useful for our purposes as well.

There are a number of open issues for future work. An important question is to get a detailed understanding of the trade-offs mentioned above for various types of searches including all sky searches for isolated GW pulsars, signals from known binary systems or from interesting areas such as the galactic center, etc. This will help us better decide how to best use our computational resources and to maximize our chances of making a detection. Another important issue, which feeds into this optimization problem, is to study the general parameter space metric. To date we only have a proper understanding of the coherent metric, i.e. case (i) above. For the other cases, we have estimates of the 
parameter space resolution and which are often sufficient for many applications, but a full understanding is still lacking. In addition, it would be interesting to compare the estimation of the amplitude parameters $\left\{A_{+}, A_{\times}\right\}$(and $\psi$ ) obtained from (6.4) and (6.5) with the maximum likelihood estimators obtained from the $\mathcal{F}$-statistic calculation. In the limit when we consider all possible correlations, we would expect the two estimates to be very close to each other.

\section{ACKNOWLEDGMENTS}

We are grateful to Stefan Ballmer for valuable discussions. We also acknowledge all members of the Continuous-Waves working group of the LIGO Scientific Collaboration for numerous discussions and suggestions which were crucial for this paper. B. K. and J.T.W. acknowledge the support of the Max-Planck-Gesellschaft. B. K. acknowledges the University of the Balearic Islands for hospitality while this work was being carried out, and the support of the DFG's special research center SFB/ Transregio 7 "Gravitational Wave Astronomy." J.T.W. also acknowledges the support of the German Aerospace Center (DLR). H. M. thanks the Council of Scientific and Industrial Research of India (CSIR) for providing a research scholarship.

Note added in proof.-Sanjeev Dhurandhar, Badri Krishnan, and John T. Whelan deeply regret the loss of their colleague and coauthor Himan Mukhopadhyay whose promising scientific career was tragically ended by her death as this paper was going to press.

\section{APPENDIX: INCLUDING SELF-CORRELATIONS AND $\mathcal{O}\left(h_{0}^{2}\right)$ CORRECTIONS}

In this section we relax the two assumptions of only looking at $\mathcal{Y}_{I J}$ for $I \neq J$ and $h \ll n$. We allow selfcorrelations (which, by themselves, are used in the standard semicoherent searches), and we keep terms of $\mathcal{O}\left(h_{0}^{2}\right)$ but still neglect $\mathcal{O}\left(h_{0}^{4}\right)$ terms.

Let us again start from the general statistic $\rho$ defined in (4.4), and let us calculate its mean and standard deviation with the two assumptions relaxed. In general, we have $\mathcal{Y}_{I J}^{*}=\mathcal{Y}_{J I}$ so that $\mathcal{Y}_{I I}$ is real and so is the corresponding weight $u_{I I} ; \mathcal{Y}_{I I}$ is in fact just the power in a single SFT bin. We will denote $\mathcal{Y}_{I I}$ simply by $\mathcal{Y}_{I}$ and $u_{I I}$ by $u_{I}$.

The mean is easy to calculate:

$$
\left\langle\mathcal{Y}_{I J}\right\rangle:=\mu_{I J}=\frac{1}{2 \Delta T} S_{n}^{I} \delta_{I J}+h_{0}^{2} \tilde{\mathcal{G}}_{I J}
$$

Thus, the mean is nonzero in the absence of a signal only for the self-correlation terms. In general, $\rho$ will contain self-correlations, and also correlations of distinct pairs. However, we want to be completely general and we do not assume that it contains all the possible pairs. This is then the expression for the mean:

$$
\langle\rho\rangle:=\mu=\frac{1}{\Delta T} \sum_{I} u_{I} S_{n}^{I}+h_{0}^{2} \sum_{\alpha}\left(u_{\alpha} \tilde{G}_{\alpha}+u_{\alpha}^{*} \tilde{G}_{\alpha}^{*}\right) .
$$

It is to be understood that the first sum in this equation only contains the self-correlations and the second sum contains all the SFT pairs we have chosen to include, including the self-correlations.

The variance calculation is somewhat more involved. Before looking at the variance of $\rho$ itself, let us look at $\left\langle\mathcal{Y}_{I J} \mathcal{y}_{K L}\right\rangle$. Note that for the pure noise terms

$$
\left\langle\tilde{n}_{I}^{*} \tilde{n}_{J} \tilde{n}_{K}^{*} \tilde{n}_{L}\right\rangle=2 \delta_{I(J} \delta_{L) K}\left\langle\left|\tilde{n}_{I}\right|^{2}\right\rangle\left\langle\left|\tilde{n}_{K}\right|^{2}\right\rangle .
$$

Here, we use the notation that indices within parentheses are symmetrized over: $X_{(I J)}=\left(X_{I J}+X_{J I}\right) / 2$. This also covers the $I=J=K=L$ case, so there is no need to consider that separately.

Consider now the signal. In general, the terms in $\left\langle\mathcal{Y}_{I J} \mathcal{Y}_{K L}\right\rangle$ with odd powers of $h$ will vanish because the noise is assumed to have zero mean. Thus, schematically, we will have

$$
\left\langle\mathcal{Y}_{I J} \mathcal{Y}_{K L}\right\rangle=A+B h_{0}^{2}+C h_{0}^{4}
$$

Let us ignore the $h_{0}^{4}$ terms and focus only on the second order terms. The reader can convince herself that we only need to keep the following terms in $\mathcal{Y}_{I J} \mathcal{Y}_{K L}$ :

$$
\tilde{h}_{I}^{*} \tilde{h}_{J} \tilde{n}_{K}^{*} \tilde{n}_{L}+\tilde{h}_{K}^{*} \tilde{h}_{J} \tilde{n}_{I}^{*} \tilde{n}_{L}+\tilde{h}_{K}^{*} \tilde{h}_{L} \tilde{n}_{I}^{*} \tilde{n}_{J}+\tilde{h}_{I}^{*} \tilde{h}_{L} \tilde{n}_{K}^{*} \tilde{n}_{J}
$$

Putting together (A3) and (A5), we end up with

$$
\begin{aligned}
\left\langle\mathcal{Y}_{I J} \mathcal{Y}_{K L}\right\rangle= & \frac{1}{2(\Delta T)^{2}} \delta_{I(J} \delta_{L) K} S_{n}^{(I)} S_{n}^{(K)} \\
& +\frac{h_{0}^{2}}{\Delta T}\left[\tilde{G}_{I(J} \delta_{L) K} S_{n}^{(K)}+\delta_{I(J} \tilde{G}_{L) K} S_{n}^{(I)}\right] .
\end{aligned}
$$

We are now ready to look at the variance of $\rho$. Let us define $\rho_{\alpha}=u_{\alpha} \mathcal{Y}_{\alpha}+u_{\alpha}^{*} \mathcal{Y}_{\alpha}^{*}$, so that $\rho=\sum_{\alpha} \rho_{\alpha}$. Then, we have

$$
\operatorname{Var}(\rho)=\sum_{\alpha} \operatorname{Var}\left(\rho_{\alpha}\right)+\sum_{\alpha, \beta(\alpha \neq \beta)} \operatorname{Cov}\left(\rho_{\alpha}, \rho_{\beta}\right) .
$$

Let us start with the variances

$$
\operatorname{Var}\left(\rho_{I J}\right)=\left\langle\rho_{I J}^{2}\right\rangle-\mu_{I J}^{2} .
$$

For $I \neq J, \mu_{I J}=\mathcal{O}\left(h_{0}^{2}\right)$ so that $\mu_{I J}^{2}$ can be ignored. Thus, in this case we get

$$
\begin{aligned}
\sigma_{\alpha}^{2} & =\operatorname{Var}\left(\rho_{I J}\right) \\
& =2\left|u_{I J}\right|^{2}\left\{\frac{S_{n}^{(I)} S_{n}^{(J)}}{4 \Delta T^{2}}+\frac{h_{0}^{2}}{2 \Delta T}\left(\tilde{G}_{I} S_{n}^{(J)}+\tilde{G}_{J} S_{n}^{(I)}\right)\right\} .
\end{aligned}
$$

For the $I=J$ case, we can no longer ignore the $\mu_{\alpha}$ term. Keeping terms up to $\mathcal{O}\left(h_{0}^{2}\right)$ we end up with

$$
\sigma_{I}^{2}=\operatorname{Var}\left(\rho_{I}\right)=4 u_{I}^{2}\left\{\left(\frac{S_{n}^{(I)}}{2 \Delta T}\right)^{2}+\frac{h_{0}^{2}}{\Delta T} \tilde{G}_{I} S_{n}^{(I)}\right\} .
$$


Turning now to the covariances, first note that if $I, J, K, L$ are all distinct, then up to $\mathcal{O}\left(h_{0}^{4}\right)$ terms, $\operatorname{Cov}\left(\rho_{I J}, \rho_{K L}\right)=0$; thus we need at least one pair of matching indices to get a nonzero result. Using (A6) the expressions for all the nonzero cases are the following $(I \neq J)$ ignoring, as always, the $\mathcal{O}\left(h_{0}^{4}\right)$ terms:

$$
\begin{aligned}
& \left\langle\mathcal{Y}_{I I} \mathcal{Y}_{I J}\right\rangle=\frac{h_{0}^{2}}{2 \Delta T}\left(\tilde{G}_{I J}+\tilde{G}_{J I}\right) S_{n}^{(I)}, \\
& \left\langle\mathcal{Y}_{I I} \mathcal{Y}_{J I}\right\rangle=\frac{h_{0}^{2}}{\Delta T} \tilde{\mathcal{G}}_{I J} S_{n}^{(I)}, \\
& \left\langle\mathcal{Y}_{I I} \mathcal{Y}_{J J}\right\rangle=\frac{S_{n}^{(I)} S_{n}^{(J)}}{4 \Delta T^{2}}+\frac{h_{0}^{2}}{2 \Delta T}\left(\tilde{G}_{I} S_{n}^{(J)}+\tilde{\mathcal{G}}_{J} S_{n}^{(I)}\right) .
\end{aligned}
$$

It turns out that the only nonzero covariance is

$$
\operatorname{Cov}\left(\rho_{I}, \rho_{I J}\right)=\frac{h_{0}^{2}}{\Delta T} u_{I} S_{n}^{(I)}\left(u_{I J} \tilde{G}_{I J}^{*}+u_{I J}^{*} \tilde{G}_{I J}\right) .
$$

We are almost done now. Substituting the results of (A9), (A10), and (A12), in (A7) we get

$$
\begin{aligned}
\sigma^{2}= & 2 \sum_{\alpha}\left|u_{\alpha}\right|^{2} \sigma_{(0), \alpha}^{2}+\frac{h_{0}^{2}}{\Delta T}\left\{\sum_{I} 4 u_{I}^{2} \tilde{G}_{I} S_{n}^{(I)}\right. \\
& +\sum_{\alpha, I \neq J}\left|u_{I J}\right|^{2}\left(\tilde{G}_{I} S_{n}^{(I)}+\tilde{G}_{J} S_{n}^{(I)}\right) \\
& \left.+\sum u_{I}\left(u_{I J} \tilde{G}_{I J}^{*}+u_{I J}^{*} \tilde{G}_{I J}\right) S_{n}^{(I)}\right\} .
\end{aligned}
$$

Here we have defined the variances in the absence of a signal:

$$
\begin{aligned}
\sigma_{(0), I} & =\frac{\left(S_{n}^{(I)}\right)^{2}}{2 \Delta T^{2}}, \\
\sigma_{(0), I J} & =\frac{S_{n}^{(I)} S_{n}^{(J)}}{4 \Delta T^{2}}, \quad I \neq J .
\end{aligned}
$$

It is convenient to write (A13) in the abbreviated form,

$$
\sigma^{2}=\sigma_{(0)}^{2}+h_{0}^{2} \sigma_{(1)}^{2},
$$

where the definitions of $\sigma_{0}$ and $\sigma_{(1)}$ are obvious from (A13).
We are finally ready to derive the equation for the sensitivity, i.e. the analogs of (4.9) and (4.15). Equation (4.7) for the threshold is unchanged as long as we use $\sigma_{(0)}$ instead of $\sigma$ in that equation. ${ }^{7}$ Equation (4.8) for the false dismissal rate becomes

$$
\gamma=\frac{1}{2} \operatorname{erfc}\left(\frac{\rho_{\mathrm{th}}-\mu}{\sqrt{2} \sigma}\right)
$$

Keeping terms linear in $h_{0}^{2}$, we get

$$
\begin{aligned}
\operatorname{erfc}^{-1}(2 \gamma)= & \frac{\rho_{\mathrm{th}}-\mu}{\sqrt{2} \sigma_{(0)}}\left(1-\frac{h_{0}^{2} \sigma_{(1)}^{2}}{2 \sigma_{(0)}^{2}}\right) \\
= & \operatorname{erfc}^{-1}(2 \alpha)-\frac{h_{0}^{2}}{\sqrt{2} \sigma_{(0)}} \sum_{\alpha}\left(u_{\alpha} \tilde{G}_{\alpha}+u_{\alpha}^{*} \tilde{G}_{\alpha}^{*}\right) \\
& -\frac{h_{0}^{2} \sigma_{(1)}^{2}}{2 \sigma_{(0)}^{2}} \operatorname{erfc}^{-1}(2 \alpha)
\end{aligned}
$$

Solving for $h_{0}$ leads to the generalization of (4.9):

$$
h_{0}^{2}=2 \mathcal{S}\left(\frac{\sqrt{\sum_{\alpha}\left|u_{\alpha}\right|^{2} \sigma_{(0), \alpha}^{2}}}{\sum_{\alpha}\left(u_{\alpha} \tilde{\mathcal{G}}_{\alpha}+u_{\alpha}^{*} \tilde{\mathcal{G}}_{\alpha}^{*}\right)+\sigma_{(1)}^{2} \operatorname{erfc}^{-1}(2 \alpha) / \sqrt{2} \sigma_{(0)}}\right) .
$$

Finding the optimal weights is now not as straightforward as before. However, we note that, when $\sigma_{(1)}$ is ignored, then the optimal weights are again given by (4.10) except that now it holds also for the self-correlations. In the general case when we do not ignore $\sigma_{(1)}$, it is simpler to continue using the optimal weights derived earlier in (4.10), and to substitute it in (A18) to derive the corresponding sensitivity.

\footnotetext{
${ }^{7}$ The mean of $\rho$ is now no longer necessarily zero in the absence of a signal (see (A2). But this only leads to an additive correction to the threshold $\rho_{\text {th }}$, and we assume this correction has been made.
}

[1] B. Abbott et al. (LIGO Scientific Collaboration), Phys. Rev. D 69, 082004 (2004).

[2] B. Abbott et al. (LIGO Scientific Collaboration), Phys. Rev. Lett. 94, 181103 (2005).

[3] B. Abbott et al. (LIGO Scientific Collaboration), Phys. Rev. D 76, 042001 (2007).

[4] B. Abbott et al. (LIGO Scientific Collaboration), Phys. Rev. D 76, 082001 (2007).

[5] B. Abbott et al. (LIGO Scientific Collaboration), Phys. Rev. D 72, 102004 (2005).
[6] B. Abbott et al. (LIGO Scientific Collaboration), Phys. Rev. D 77, 022001 (2008).

[7] B. Abbott et al. (LIGO Scientific Collaboration), Phys. Rev. D 76, 082003 (2007).

[8] P. Astone et al., arXiv:0708.4367.

[9] P. R. Brady and T. Creighton, Phys. Rev. D 61, 082001 (2000).

[10] V. Dergachev and K. Riles (LIGO Scientific Collaboration), Report No. LIGO-T050186-00-Z, 2005, http://admdbsrv.ligo.caltech.edu/dcc. 
[11] B. Krishnan et al., Phys. Rev. D 70, 082001 (2004).

[12] C. Cutler, I. Gholami, and B. Krishnan, Phys. Rev. D 72, 042004 (2005).

[13] http://einstein.phys.uwm.edu.

[14] L. Bildsten, Astrophys. J. Lett. 501, L89 (1998).

[15] B. Haskell, N. Andersson, D. I. Jones, and L. Samuelsson, arXiv:0708.2984.

[16] B. J. Owen, Phys. Rev. Lett. 95, 211101 (2005).

[17] C. Cutler and B.F. Schutz, Phys. Rev. D 72, 063006 (2005).

[18] S. W. Ballmer, Classical Quantum Gravity 23, S179 (2006).

[19] B. Allen and J.D. Romano, Phys. Rev. D 59, 102001 (1999).

[20] S. Mitra et al., Phys. Rev. D 77, 042002 (2008).

[21] B. Abbott et al. (LIGO Scientific Collaboration), Phys. Rev. D 69, 122004 (2004).

[22] B. Abbott et al. (LIGO Scientific Collaboration), Phys. Rev. Lett. 95, 221101 (2005).

[23] B. Abbott et al. (LIGO Scientific Collaboration), Astrophys. J. 659, 918 (2007).

[24] P. Jaranowski, A. Krolak, and B. F. Schutz, Phys. Rev. D 58, 063001 (1998).

[25] S. V. Dhurandhar and A. Vecchio, Phys. Rev. D 63, 122001 (2001).

[26] E. L. Wright, E. W. Gottlieb, and W. Liller, Astrophys. J. 200, 171 (1975).
[27] D. Steeghs and J. Casares, Astrophys. J. 568, 273 (2002).

[28] B. Allen and G. Mendell (LIGO Scientific Collaboration), Report No. LIGO-T040164-01-Z, 2004, http://admdbsrv. ligo.caltech.edu/dcc.

[29] D. Percival and A. Walden, Spectral Analysis for Physical Applications (Cambridge University Press, Cambridge, England, 1993).

[30] J. Whelan (LIGO Scientific Collaboration), Report No. LIGO-T060162-01-Z, 2006, http://admdbsrv.ligo. caltech.edu/dcc.

[31] J. T. Whelan, Classical Quantum Gravity 23, 1181 (2006).

[32] B. Krishnan and A. Sintes (LIGO Scientific Collaboration), Report No. LIGO-T070124-00-Z, 2007, http://admdbsrv.ligo.caltech.edu/dcc.

[33] W. Feller, An Introduction to Probability Theory and its Applications (Wiley, New York, 1965), Vol. II.

[34] R. Prix, Phys. Rev. D 75, 023004 (2007).

[35] G. Mendell and K. Wette, arXiv:0710.4362.

[36] B. S. Sathyaprakash and S. V. Dhurandhar, Phys. Rev. D 44, 3819 (1991).

[37] S. V. Dhurandhar and B. S. Sathyaprakash, Phys. Rev. D 49, 1707 (1994).

[38] B. J. Owen, Phys. Rev. D 53, 6749 (1996).

[39] P. R. Brady, T. Creighton, C. Cutler, and B. F. Schutz, Phys. Rev. D 57, 2101 (1998).

[40] L. Wen, X.-1. Fan, and Y. Chen, arXiv:0711.0726. 